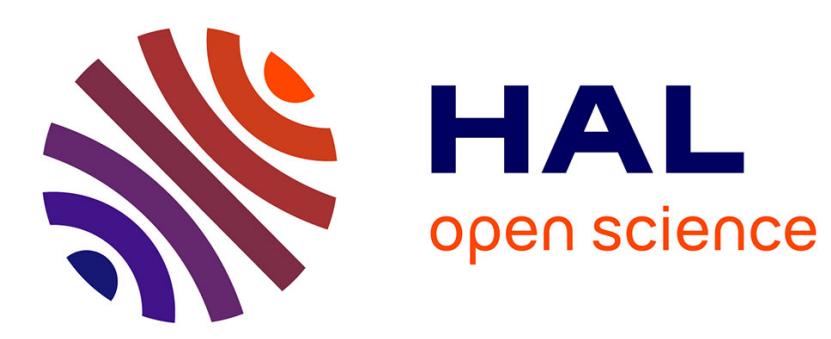

\title{
Gevrey order and summability of formal series solutions of certain classes of inhomogeneous linear integro-differential equations with variable coefficients
} Pascal Remy

\section{- To cite this version: \\ Pascal Remy. Gevrey order and summability of formal series solutions of certain classes of inhomoge- neous linear integro-differential equations with variable coefficients. 2016. hal-01267736}

\author{
HAL Id: hal-01267736 \\ https://hal.science/hal-01267736
}

Preprint submitted on 4 Feb 2016

HAL is a multi-disciplinary open access archive for the deposit and dissemination of scientific research documents, whether they are published or not. The documents may come from teaching and research institutions in France or abroad, or from public or private research centers.
L'archive ouverte pluridisciplinaire HAL, est destinée au dépôt et à la diffusion de documents scientifiques de niveau recherche, publiés ou non, émanant des établissements d'enseignement et de recherche français ou étrangers, des laboratoires publics ou privés. 


\title{
Gevrey order and summability of formal series solutions of certain classes of inhomogeneous linear integro-differential equations with variable coefficients
}

\author{
Pascal Remy \\ Lycée Les Pierres Vives \\ 1 rue des Alouettes \\ F-78 420 Carrières-sur-Seine, France \\ email: pascal.remy07@orange.fr
}

\begin{abstract}
In this article, we investigate Gevrey and summability properties of formal power series solutions of certain classes of inhomogeneous linear integro-differential equations with analytic coefficients in a neighborhood of $(0,0) \in \mathbb{C}^{2}$. In particular, we give necessary and sufficient conditions under which these solutions are convergent or are $k$-summable, for a convenient positive rational number $k$, in a given direction.
\end{abstract}

Keywords. Linear integro-differential equation, divergent power series, Newton polygon, Gevrey order, summability

AMS subject classification. $35 \mathrm{C} 10,35 \mathrm{C} 20,40 \mathrm{~B} 05$

\section{Setting the problem}

For several years, various works allowed to formulate many results on Gevrey properties, summability and multisummability of divergent solutions of some linear partial differential equations or linear integro-differential equations with constant coefficients (see $[1,3,6,7,16,25]$ etc.) or variable coefficients (see $[5,10-$ $13,17-19,23,24,27-29,32-34]$ etc.) in two variables or more.

In this paper, we are interested in inhomogeneous linear integro-differential equations of the form

$$
D u=\widetilde{f}(t, x) \quad, D:=1-P\left(\partial_{t}^{-1}, \partial_{x}\right)
$$

where $P(T, X)=\sum_{i \in \mathcal{K} q=0} \sum_{i}^{p_{i}} a^{(i, q)}(t, x) T^{i} X^{q} \in \mathcal{O}\left(D_{\rho_{1}} \times D_{\rho_{2}}\right)[T, X]$ is a nonzero polynomial in two variables $T$ and $X$ satisfying the following conditions

$\left(C_{1}\right) \mathcal{K}$ is a nonempty finite subset of $\mathbb{N}^{*}$ (= the set of positive integers),

$\left(C_{2}\right) p_{i} \geq 0$ is a nonnegative integer for all $i \in \mathcal{K}$, 
$\left(C_{3}\right)$ the coefficients $a^{(i, q)}(t, x)$ are holomorphic in the two variables $t$ and $x$ in a polydisc $D_{\rho_{1}} \times D_{\rho_{2}}$ centered at the origin $(0,0) \in \mathbb{C}^{2}\left(D_{\rho_{j}}\right.$ denotes the disc with center 0 and radius $\left.\rho_{j}>0\right)$ for all $i \in \mathcal{K}$ and $q \in\left\{0, \ldots, p_{i}\right\}$,

$\left(C_{4}\right) a^{\left(i, p_{i}\right)}(0, x) \not \equiv 0$ for all $i \in \mathcal{K}$.

and where $\tilde{f}(t, x) \in \mathcal{O}\left(D_{\rho_{2}}\right)[[t]]^{1}$ may be smooth or not. Notation $\partial_{t}^{-1} u$ stands for the anti-derivation $\int_{0}^{t} u(s, x) d s$ of $u$ with respect to $t$ which vanishes at $t=0$.

Notation 1.1 For any series $\widetilde{u}(t, x) \in \mathcal{O}\left(D_{\rho_{2}}\right)[[t]]$, we denote in the sequel

$$
\widetilde{u}(t, x)=\sum_{j \geq 0} u_{j, *}(x) \frac{t^{j}}{j !}=\sum_{n \geq 0} \widetilde{u}_{*, n}(t) \frac{x^{n}}{n !}=\sum_{j, n \geq 0} u_{j, n} \frac{t^{j}}{j !} \frac{x^{n}}{n !}
$$

Under conditions above, it is easy to check that equation (1.1) has a unique solution in $\mathcal{O}\left(D_{\rho_{2}}\right)[[t]]$. More precisely, we have the following.

Theorem 1.2 $D$ is a linear automorphism of $\mathcal{O}\left(D_{\rho_{2}}\right)[[t]]$.

Proof. Let us begin by observing that $D$ is a linear operator acting inside $\mathcal{O}\left(D_{\rho_{2}}\right)[[t]]$. Indeed, $\left(\mathcal{O}\left(D_{\rho_{2}}\right)[[t]], \partial_{t}, \partial_{x}\right)$ is a $\mathbb{C}$-differential algebra stable under anti-derivation $\partial_{t}^{-1}$ (and anti-derivation $\partial_{x}^{-1}$ too) and the coefficients $a^{(i, q)}(t, x)$ belong to $\mathcal{O}\left(D_{\rho_{1}} \times D_{\rho_{2}}\right) \subset \mathcal{O}\left(D_{\rho_{2}}\right)[[t]]$ for all $i$ and $q$. On the other hand, given $\tilde{f}(t, x) \in \mathcal{O}\left(D_{\rho_{2}}\right)[[t]]$, a series $\widetilde{u}(t, x)=\sum_{j \geq 0} u_{j, *}(x) \frac{t^{j}}{j !}$ is a solution of $D u=\tilde{f}(t, x)$ if and only if its coefficients $u_{j, *}(x)$ satisfy, for all $j \geq 0$, the identities

$$
u_{j, *}(x)=f_{j, *}(x)+\sum_{i \in \mathcal{K}} \sum_{q=0}^{p_{i}} \sum_{m=0}^{j-i}\left(\begin{array}{c}
j \\
m
\end{array}\right) a_{m, *}^{(i, q)}(x) \partial_{x}^{q} u_{j-m-i, *}(x)
$$

with the classical convention that the third sum is 0 if $j<i$. Thereby, equation $D u=\widetilde{f}(t, x)$ admits a unique solution $\widetilde{u}(t, x) \in \mathcal{O}\left(D_{\rho_{2}}\right)[[t]]$, which proves the bijectivity of $D$ and ends the proof.

Corollary 1.3 Equation (1.1) admits a unique formal series solution $\widetilde{u}(t, x) \in$ $\mathcal{O}\left(D_{\rho_{2}}\right)[[t]]$. Moreover, its coefficients $u_{j, *}(x) \in \mathcal{O}\left(D_{\rho_{2}}\right)$ are recursively determined for all $j \geq 0$ by identities (1.2).

Note that $\widetilde{u}(t, x)$ is divergent in general.

Remark 1.4 Let $\kappa \in \mathbb{N}^{*}$ and $\mathcal{K}$ a nonempty subset of $\{1, \ldots, \kappa\}$. Let the Cauchy problem

$$
\left\{\begin{array}{l}
\left(\partial_{t}^{\kappa}-\sum_{i \in \mathcal{K}} \sum_{i=0}^{p_{i}} a^{(i, q)}(t, x) \partial_{t}^{\kappa-i} \partial_{x}^{q}\right) U=\widetilde{q}(t, x) \\
\partial_{t}^{j} U(t, x)_{\mid t=0}=\varphi_{j}(x) \quad, j=0, \ldots, \kappa-1
\end{array}\right.
$$

${ }^{1}$ We denote $\tilde{f}$ with a tilde to emphasize the possible divergence of the series $\tilde{f}$. 
with inhomogeneity $\widetilde{q}(t, x) \in \mathcal{O}\left(D_{\rho_{2}}\right)[[t]]$ and initial conditions $\varphi_{j}(x) \in \mathcal{O}\left(D_{\rho_{2}}\right)$ for all $j$. Then, the change of unknown function $U$ to $u$ by

$$
U(t, x)=\sum_{j=0}^{\kappa-1} \varphi_{j}(x) \frac{t^{j}}{j !}+\partial_{t}^{-\kappa} u(t, x)
$$

allows to reduce problem (1.3) to equation (1.1) with

$$
\widetilde{f}(t, x)=\widetilde{q}(t, x)-\sum_{i \in \mathcal{K}} \sum_{q=0}^{p_{i}} \sum_{j=\kappa-i}^{\kappa-1} a^{(i, q)}(t, x) \partial_{x}^{q} \varphi_{j}(x) \frac{t^{j-\kappa+i}}{(j-\kappa+i) !} .
$$

More precisely, it is easy to check that the unique solvability in $\mathcal{O}\left(D_{\rho_{2}}\right)[[t]]$ of equation (1.1) (which is proved in theorem 1.2 above) is equivalent to the unique solvability in $\mathcal{O}\left(D_{\rho_{2}}\right)[[t]]$ of problem (1.3). Besides, these two solutions have the same Gevrey and summability/multisummability properties since $\varphi_{0}(x)+$ $\varphi_{1}(x) t+\ldots+\varphi_{\kappa-1}(x) t^{\kappa-1} /(\kappa-1) !$ is analytic at the origin $(0,0) \in \mathbb{C}^{2}$. Thereby, it is equivalent to work with the Cauchy problem (1.3) or with the integrodifferential equation (1.1). For both calculations and notational conveniences, it is this latter point of view we have chosen to adopt here.

In this article, we propose to study some Gevrey and summability properties related to the unique formal solution $\widetilde{u}(t, x)$ of equation (1.1). Denoting $\mathcal{I}_{\mathcal{K}}:=\left\{i \in \mathcal{K} ; p_{i}>i\right\}$, we first show in section 3 that $\widetilde{u}(t, x)$ and the inhomogeneity $\tilde{f}(t, x)$ are together convergent when $\mathcal{I}_{\mathcal{K}}=\emptyset$ and $1 / k$-Gevrey, with $k$ the smallest positive slope of the Newton polygon at $t=0$ of $D$, otherwise. Then, in this latter case, and under two additionnal conditions on $D$, we investigate the summability of $\widetilde{u}(t, x)$. In particular, we prove in section 4 , through a fixed point method, a necessary and sufficient condition under which $\widetilde{u}(t, x)$ is $k$-summable in a given direction $\arg (t)=\theta$.

\section{Gevrey order and summability of formal series}

In this section, we recall, for the convenience of the reader, some definitions and basic properties about the Gevrey order and the summability of formal power series in $\mathcal{O}\left(D_{\rho_{2}}\right)[[t]]$, which are needed in the sequel.

All along the article, we consider $t$ as the variable and $x$ as a parameter. Doing that, any formal power series $\widetilde{u}(t, x) \in \mathcal{O}\left(D_{\rho_{2}}\right)[[t]]$ can be seen as a formal power series in $t$ with coefficients in the Banach space $\mathcal{O}\left(D_{\rho_{2}}\right)$. Thereby, to define the notions of Gevrey classes and summability of such formal series, one extends the classical notions of Gevrey classes and summability of elements in $\mathbb{C}[[t]]$ to families parametrized by $x$ in requiring similar conditions, the estimates being however uniform with respect to $x$. For a general treatment of this theory, we refer for instance to [2].

\section{$2.1 \quad s$-Gevrey formal series}

The classical notion of $s$-Gevrey formal series is extended to $x$-families as follows. 
Definition 2.1 Let $s \geq 0$. A series $\widetilde{u}(t, x)=\sum_{j \geq 0} u_{j, *}(x) \frac{t^{j}}{j !} \in \mathcal{O}\left(D_{\rho_{2}}\right)[[t]]$ is said to be Gevrey of order $s$ (in short, $s$-Gevrey) if there exist $0<r_{2} \leq \rho_{2}, C>0$ and $K>0$ such that inequalities

$$
\left|u_{j, *}(x)\right| \leq C K^{j} \Gamma(1+(s+1) j)
$$

hold for all $j \geq 0$ and $x \in D_{r_{2}}$.

In other words, definition 2.1 means that $\widetilde{u}(t, x)$ is $s$-Gevrey in $t$, uniformly in $x$ on a neighborhood of $x=0$.

We denote by $\mathcal{O}\left(D_{\rho_{2}}\right)[[t]]_{s}$ the set of all the formal series in $\mathcal{O}\left(D_{\rho_{2}}\right)[[t]]$ which are $s$-Gevrey. Note that the set $\mathcal{O}\left(D_{\rho_{2}}\right)[[t]]_{0}$ coincides with the set $\mathbb{C}\{t, x\}$ of germs of analytic functions at the origin $(0,0) \in \mathbb{C}^{2}$. Note also that the sets $\mathcal{O}\left(D_{\rho_{2}}\right)[[t]]_{s}$ are filtered as follows:

$$
\mathbb{C}\{t, x\}=\mathcal{O}\left(D_{\rho_{2}}\right)[[t]]_{0} \subset \mathcal{O}\left(D_{\rho_{2}}\right)[[t]]_{s} \subset \mathcal{O}\left(D_{\rho_{2}}\right)[[t]]_{s^{\prime}} \subset \mathcal{O}\left(D_{\rho_{2}}\right)[[t]]
$$

for all $s$ and $s^{\prime}$ satisfying $0<s<s^{\prime}<+\infty$.

Following proposition 2.2 precises the algebraic structure of the $\mathcal{O}\left(D_{\rho_{2}}\right)[[t]]_{s}$ 's.

Proposition 2.2 Let $s \geq 0$. Then, $\left(\mathcal{O}\left(D_{\rho_{2}}\right)[[t]]_{s}, \partial_{t}, \partial_{x}\right)$ is a $\mathbb{C}$-differential algebra stable under anti-derivations $\partial_{t}^{-1}$ and $\partial_{x}^{-1}$.

Proof. See for instance [32, Prop. 1] or [2, p. 64].

Let us now define the notion of $k$-summability of a series $\widetilde{u}(t, x) \in \mathcal{O}\left(D_{\rho_{2}}\right)[[t]]$ at $t=0$.

\section{$2.2 k$-summability}

Among the many equivalent definitions of $k$-summability in a given direction $\arg (t)=\theta$ at $t=0$, we choose in this article a generalization of Ramis' definition which states that a formal series $\widetilde{g}(t, x) \in \mathbb{C}[[t]]$ is $k$-summable in direction $\theta$ if there exists a holomorphic function $g$ which is $1 / k$-Gevrey asymptotic to $\widetilde{g}$ in an open sector $\Sigma_{\theta,>\pi / k}$ bisected by $\theta$ and with opening larger than $\pi / k$ [30, Def. 3.1 . To express the $1 / k$-Gevrey asymptotic, there also exist various equivalent ways. We choose here the one which sets conditions on the successive derivatives of $g$ (see [20, p. 171] or [30, Thm. 2.4] for instance).

Definition 2.3 ( $k$-summability) Let $k>0$ and $s=1 / k$. A formal series $\widetilde{u}(t, x) \in \mathcal{O}\left(D_{\rho_{2}}\right)[[t]]$ is said to be $k$-summable in the direction $\arg (t)=\theta$ if there exist a sector $\Sigma_{\theta,>\pi s}$, a radius $0<r_{2} \leq \rho_{2}$ and a function $u(t, x)$ called $k$-sum of $\widetilde{u}(t, x)$ in direction $\theta$ such that

1. $u$ is defined and holomorphic on $\Sigma_{\theta,>\pi s} \times D_{r_{2}}$;

2. For any $x \in D_{r_{2}}$, the map $t \mapsto u(t, x)$ has $\widetilde{u}(t, x)=\sum_{j \geq 0} u_{j, *}(x) \frac{t^{j}}{j !}$ as Taylor series at 0 on $\Sigma_{\theta,>\pi s}$;

3. For any proper ${ }^{2}$ subsector $\Sigma \Subset \Sigma_{\theta,>\pi s}$, there exist constants $C>0$ and $K>0$ such that, for all $\ell \geq 0$, all $t \in \Sigma$ and all $x \in D_{r_{2}}$,

\footnotetext{
${ }^{2}$ A subsector $\Sigma$ of a sector $\Sigma^{\prime}$ is said to be a proper subsector and one denotes $\Sigma \Subset \Sigma^{\prime}$ if its closure in $\mathbb{C}$ is contained in $\Sigma^{\prime} \cup\{0\}$.
} 


$$
\left|\partial_{t}^{\ell} u(t, x)\right| \leq C K^{\ell} \Gamma(1+(s+1) \ell) .
$$

We denote by $\mathcal{O}\left(D_{\rho_{2}}\right)\{t\}_{k ; \theta}$ the subset of $\mathcal{O}\left(D_{\rho_{2}}\right)[[t]]$ made of all the $k$-summable formal series in the direction $\arg (t)=\theta$. Obviously, we have $\mathcal{O}\left(D_{\rho_{2}}\right)\{t\}_{k ; \theta} \subset$ $\mathcal{O}\left(D_{\rho_{2}}\right)[[t]]_{s}$.

Note that, for any fixed $x \in D_{r_{2}}$, the $k$-summability of $\widetilde{u}(t, x)$ coincides with the classical $k$-summability. Consequently, Watson's lemma implies the unicity of its $k$-sum, if any exists.

Note also that the $k$-sum of a $k$-summable formal series $\widetilde{u}(t, x) \in \mathcal{O}\left(D_{\rho_{2}}\right)\{t\}_{k ; \theta}$ may be analytic with respect to $x$ on a disc $D_{r_{2}}$ smaller than the common disc $D_{\rho_{2}}$ of analyticity of the coefficients $u_{j, *}(x)$ of $\widetilde{u}(t, x)$.

Obsviously, the set $\mathcal{O}\left(D_{\rho_{2}}\right)\{t\}_{k ; \theta}$ is a subspace of $\mathcal{O}\left(D_{\rho_{2}}\right)[[t]]_{s}$. Proposition 2.4 below precises its algebraic structure.

Proposition 2.4 Let $k>0$ and $\theta \in \mathbb{R} / 2 \pi \mathbb{Z}$. Then, $\left(\mathcal{O}\left(D_{\rho_{2}}\right)\{t\}_{k ; \theta}, \partial_{t}, \partial_{x}\right)$ is a $\mathbb{C}$-differential algebra stable under anti-derivatives $\partial_{t}^{-1}$ and $\partial_{x}^{-1}$.

Proof. It is sufficient to prove that $\mathcal{O}\left(D_{\rho_{2}}\right)\{t\}_{k ; \theta}$ is stable under multiplication, derivations and anti-derivations.

$\triangleleft$ Multiplication. Let $\widetilde{u}(t, x), \widetilde{v}(t, x) \in \mathcal{O}\left(D_{\rho_{2}}\right)\{t\}_{k ; \theta}$ be two $k$-summable formal series in direction $\theta$ with $k$-sums $u(t, x)$ and $v(t, x)$ and $\widetilde{w}=\widetilde{u v}$. In definition 2.3 above, we can always choose the same constants $r_{2}, C$ and $K$ and the same sector $\Sigma_{\theta,>\pi s}$ both for $\widetilde{u}$ and $\widetilde{v}$. Obviously, the product $w(t, x)=u(t, x) v(t, x)$ satisfies conditions 1 and 2 of definition 2.3. Moreover, given a proper subsector $\Sigma \Subset \Sigma_{\theta,>\pi s}$ and using Leibniz formula, we get, for all $\ell \geq 0, t \in \Sigma$ and $x \in D_{r_{2}}$,

$$
\begin{aligned}
\left|\partial_{t}^{\ell} w(t, x)\right| & \leq \sum_{j=0}^{\ell}\left(\begin{array}{l}
\ell \\
j
\end{array}\right)\left|\partial_{t}^{j} u(t, x)\right|\left|\partial_{t}^{\ell-j} v(t, x)\right| \\
& \leq C^{2} K^{\ell} \sum_{j=0}^{\ell}\left(\begin{array}{l}
\ell \\
j
\end{array}\right) \underbrace{\Gamma(1+(s+1) j) \Gamma(1+(s+1)(\ell-j))}_{a_{\ell, j}}
\end{aligned}
$$

where, according to relations between the Gamma and Beta functions,

$$
a_{\ell, j}=\Gamma(2+(s+1) \ell) \int_{0}^{1} t^{(s+1) j}(1-t)^{(s+1)(\ell-j)} d t \leq \Gamma(2+(s+1) \ell .
$$

Thereby,

$$
\begin{aligned}
\left|\partial_{t}^{\ell} w(t, x)\right| & \leq C^{2} K^{\ell} \Gamma(2+(s+1) \ell) \sum_{j=0}^{\ell}\left(\begin{array}{l}
\ell \\
j
\end{array}\right) \\
& =C^{2}(2 K)^{\ell}(1+(s+1) \ell) \Gamma(1+(s+1) \ell) \\
& \leq C^{2}(2 K)^{\ell} e^{1+(s+1) \ell} \Gamma(1+(s+1) \ell)
\end{aligned}
$$

and, consequently,

$$
\left|\partial_{t}^{\ell} w(t, x)\right| \leq C^{\prime} K^{\prime \ell} \Gamma(1+(s+1) \ell)
$$


with $C^{\prime}=e C^{2}$ et $K^{\prime}=2 K e^{s+1}$. This proves condition 3 of definition 2.3; hence the stability of $\mathcal{O}\left(D_{\rho_{2}}\right)\{t\}_{k ; \theta}$ under multiplication.

$\triangleleft$ The stability of $\mathcal{O}\left(D_{\rho_{2}}\right)\{t\}_{k ; \theta}$ under derivation $\partial_{t}$ and under anti-derivations $\partial_{t}^{-1}$ and $\partial_{x}^{-1}$ is straightforward and is left to the reader.

$\triangleleft$ Derivation $\partial_{x}$. Let $\widetilde{u}(t, x) \in \mathcal{O}\left(D_{\rho_{2}}\right)\{t\}_{k ; \theta}$ and $\widetilde{w}(t, x)=\partial_{x} \widetilde{u}(t, x)$. Let $u(t, x)$ be the $k$-sum of $\widetilde{u}(t, x)$. Then, $w(t, x)=\partial_{x} u(t, x)$ satisfies conditions 1 and 2 of definition 2.3. Let us now fix a proper subsector $\Sigma \Subset \Sigma_{\theta,>\pi s}$ and choose a sector $\Sigma^{\prime}$ such that $\Sigma \Subset \Sigma^{\prime} \Subset \Sigma_{\theta,>\pi s}$. By assumption, there exist $C, K>0$ such that $\left|\partial_{t}^{\ell} u(t, x)\right| \leq C K^{\ell} \Gamma(1+(s+1) \ell)$ for all $\ell \geq 0, t \in \Sigma^{\prime}$ and $x \in D_{r_{2}}$. Let $\delta$ be so small that, for all $t \in \Sigma$, the closed disc with center $t$ and radius $|t| \delta$ be contained in $\Sigma^{\prime}$. Then, denoting $u_{\ell}(t, x)=\partial_{t}^{\ell} u(t, x)$ and choosing $0<r_{2}^{\prime}<r_{2}$, Cauchy integral formula yields relation

$$
\partial_{t}^{\ell} w(t, x)=\partial_{x} u_{\ell}(t, x)=\frac{1}{(2 i \pi)^{2}} \int_{\substack{\left|t^{\prime}-t\right|=|t| \delta \\\left|x^{\prime}-x\right|=r_{2}-r_{2}^{\prime}}} \frac{u_{\ell}\left(t^{\prime}, x^{\prime}\right)}{\left(t^{\prime}-t\right)\left(x^{\prime}-x\right)^{2}} d t^{\prime} d x^{\prime} ;
$$

hence, the inequality

$$
\begin{aligned}
\left|\partial_{t}^{\ell} w(t, x)\right| & \leq \frac{1}{r_{2}-r_{2}^{\prime}} \sup _{\left(t^{\prime}, x^{\prime}\right) \in \Sigma^{\prime} \times D_{r_{2}}}\left|u_{\ell}\left(t^{\prime}, x^{\prime}\right)\right| \\
& \leq C^{\prime} K^{\ell} \Gamma(1+(s+1) \ell) \quad \text { with } C^{\prime}=\frac{C}{r_{2}-r_{2}^{\prime}}
\end{aligned}
$$

for all $\ell \geq 0, t \in \Sigma$ and $x \in D_{r_{2}^{\prime}}$. This proves condition 3 of definition 2.3 and, consequently, the stability of $\mathcal{O}\left(D_{\rho_{2}}\right)\{t\}_{k ; \theta}$ under derivation $\partial_{x}$.

With respect to $t$, the $k$-sum $u(t, x)$ of a $k$-summable series $\widetilde{u}(t, x) \in \mathcal{O}\left(D_{\rho_{2}}\right)\{t\}_{k ; \theta}$ is analytic on an open sector for which there is no control on the angular opening except that it must be larger than $\pi / k$ (hence, it contains a closed sector $\bar{\Sigma}_{\theta, \pi / k}$ bisected by $\theta$ and with opening $\pi / k)$ and no control on the radius except that it must be positive. Thereby, the $k$-sum $u(t, x)$ is well-defined as a section of the sheaf of analytic functions in $(t, x)$ on a germ of closed sector of opening $\pi / k$ (i.e., a closed interval $\bar{I}_{\theta, \pi / k}$ of length $\pi / k$ on the circle $S^{1}$ of directions issuing from 0 ; see $[21,1.1]$ or $[14$, I.2]) times $\{0\}$ (in the plane $\mathbb{C}$ of the variable $x$ ). We denote by $\mathcal{O}_{\bar{I}_{\theta, \pi / k} \times\{0\}}$ the space of such sections.

Corollary 2.5 The operator of k-summation

$$
\begin{aligned}
& \mathcal{S}_{k ; \theta}: \mathcal{O}\left(D_{\rho_{2}}\right)\{t\}_{k ; \theta} \longrightarrow \mathcal{O}_{\bar{I}_{\theta, \pi / k} \times\{0\}} \\
& \widetilde{u}(t, x) \quad \longmapsto u(t, x)
\end{aligned}
$$

is a homomorphism of differential $\mathbb{C}$-algebras for the derivations $\partial_{t}$ and $\partial_{x}$. Moreover, it commutes with the anti-derivations $\partial_{t}^{-1}$ and $\partial_{x}^{-1}$.

Let us now turn to the study of the unique formal series solution $\widetilde{u}(t, x) \in$ $\mathcal{O}\left(D_{\rho_{2}}\right)[[t]]$ of equation (1.1).

\section{Gevrey properties of $\widetilde{u}(t, x)$}

The aim of this section is to investigate Gevrey properties of $\widetilde{u}(t, x)$. In particular, we propose to give necessary and sufficient conditions under which $\widetilde{u}(t, x)$ 
is $s$-Gevrey for some $s \geq 0$. Before starting the calculations, let us define the Newton polygon of $D$ at $t=0$.

\subsection{Newton polygon}

As definition of the Newton polygon, we choose the definition of M. Miyake [24] (see also A. Yonemura [34] or S. Ouchi [27]) which is an analogue to the one given by J.-P. Ramis [31] for linear ordinary differential equations. Recall that, H. Tahara and H. Yamazawa use in [33] a slightly different one.

For any $(a, b) \in \mathbb{R}^{2}$, we denote by $C(a, b)$ the domain

$$
C(a, b)=\left\{(x, y) \in \mathbb{R}^{2} ; x \leq a \text { and } y \geq b\right\} .
$$

For any formal series $a(t, x) \in \mathcal{O}\left(D_{\rho_{2}}\right)[[t]]$, we also denote by $v_{a l}(a)$ the valuation of $a(t, x)$ with respect to $t$, i.e., the order of the zero of $a(t, x)$ at $t=0$.

Definition 3.1 The Newton polygon $N_{t}(D)$ of $D$ at $t=0$ is defined as the convex hull of the union of sets $C(0,0)$ and $C\left(q-i, v_{a l}\left(a^{(i, q)}\right)+i\right)$ for $i \in \mathcal{K}$ and $q \in\left\{0, \ldots, p_{i}\right\}$ :

$$
N_{t}(D)=C H\left[C(0,0) \cup \bigcup_{\substack{i \in \mathcal{K} \\ q \in\left\{0, \ldots, p_{i}\right\}}} C\left(q-i, v^{2} a_{t}\left(a^{(i, q)}\right)+i\right)\right]
$$

where $C H[\cdot]$ denotes the convex hull of the elements in [·].

Following lemma 3.2 gives us some properties of $N_{t}(D)$.

Lemma 3.2 Let $\mathcal{I}_{\mathcal{K}}:=\left\{i \in \mathcal{K} ; p_{i}>i\right\}$.

1. Assume $\mathcal{I}_{\mathcal{K}}=\emptyset$. Then, $N_{t}(D)=C(0,0)$. In particular, $N_{t}(D)$ has no side with a positive slope.

2. Assume $\mathcal{I}_{\mathcal{K}} \neq \emptyset$. Then, $N_{t}(D)$ has (at least) one side with a positive slope. Moreover, its smallest positive slope $k$ is given by

$$
k=\min \left(\frac{i}{p_{i}-i} ; i \in \mathcal{I}_{\mathcal{K}}\right)
$$

and the side of slope $k$ has for length $p_{i_{0}}-i_{0}$, where

$$
i_{0}=\max \left(i \in \mathcal{K} ; \frac{i}{p_{i}-i}=k\right) .
$$

Proof. Point 1 stems obvious from the fact that condition $\mathcal{I}_{\mathcal{K}}=\emptyset$ implies $C\left(q-i, v_{a l}\left(a^{(i, q)}\right)+i\right) \subset C(0,0)$ for all $i$ and $q$. As for point 2 , it suffices to remark, on one hand, that condition $\left(C_{4}\right)$ implies $C\left(q-i, v a l_{t}\left(a^{(i, q)}\right)+i\right) \subset$ $C\left(p_{i}-i, i\right)$ for all $i, q$ and, on the other hand, that the segment with two end points $(0,0)$ and $\left(p_{i}-i, i\right)$ has a slope equal to $i /\left(p_{i}-i\right)$ which is positive if and only if $i \in \mathcal{I}_{\mathcal{K}}$. 
In the sequel, we set $k:=\frac{i_{0}}{p_{i_{0}}-i_{0}}$ when $\mathcal{I}_{\mathcal{K}} \neq \emptyset$. Note that the following inequalities

$$
\frac{p_{i_{0}}}{i_{0}} \geq \frac{p_{i}}{i}
$$

hold for all $i \in \mathcal{K}$.

Let us now turn to the Gevrey properties of $\widetilde{u}(t, x)$.

\subsection{Gevrey order}

Let us begin by observing that proposition 2.2 implies the following.

Lemma 3.3 $D\left(\mathcal{O}\left(D_{\rho_{2}}\right)[[t]]_{s}\right) \subset \mathcal{O}\left(D_{\rho_{2}}\right)[[t]]_{s}$ for all $s \geq 0$.

Main theorem 3.4 below precises this statement by showing more especially that $D$ is actually a linear automorphism of $\mathcal{O}\left(D_{\rho_{2}}\right)[[t]]_{s}$ for some $s \geq 0$.

Theorem 3.4 Let $\mathcal{I}_{\mathcal{K}}:=\left\{i \in \mathcal{K} ; p_{i}>i\right\}$ and s the rational number defined by

$$
s:=\left\{\begin{array}{ll}
0 & \text { if } \mathcal{I}_{\mathcal{K}}=\emptyset \\
1 / k=p_{i_{0}} / i_{0}-1 & \text { if } \mathcal{I}_{\mathcal{K}} \neq \emptyset
\end{array} .\right.
$$

Then, $D$ is a linear automorphism of $\mathcal{O}\left(D_{\rho_{2}}\right)[[t]]_{s}$.

In particular, theorem 3.4 gives us Gevrey properties of $\widetilde{u}(t, x)$ in view in this section. More precisely, it provides, in the case $\mathcal{I}_{\mathcal{K}}=\emptyset$, necessary and sufficient condition under which $\widetilde{u}(t, x)$ is convergent and, in the opposite case $\mathcal{I}_{\mathcal{K}} \neq \emptyset$, necessary and sufficient condition under which $\widetilde{u}(t, x)$ is $s$-Gevrey with $s=p_{i_{0}} / i_{0}-1$.

Corollary 3.5 Let $\mathcal{I}_{\mathcal{K}}:=\left\{i \in \mathcal{K} ; p_{i}>i\right\}$.

1. Assume $\mathcal{I}_{\mathcal{K}}=\emptyset$. Then, $\widetilde{u}(t, x)$ is convergent if and only if the inhomogeneity $\widetilde{f}(t, x)$ is convergent.

2. Assume $\mathcal{I}_{\mathcal{K}} \neq \emptyset$ and let $s=p_{i_{0}} / i_{0}-1$. Then, $\widetilde{u}(t, x)$ is $s$-Gevrey if and only if the inhomogeneity $\widetilde{f}(t, x)$ is s-Gevrey.

Note that point 1 can be actually extended to any integro-differential equation of the form (1.1), where condition $\left(C_{4}\right)$ fails, but is replaced by the very general condition $a^{\left(i, p_{i}\right)} \not \equiv 0$ for all $i \in \mathcal{K}$. We shall come back later to this (see remark 3.10). For the moment, let us prove the main theorem 3.4.

\subsection{Proof of theorem 3.4}

According to theorem 1.2 and lemma 3.3, $D$ is an injective linear operator acting inside $\mathcal{O}\left(D_{\rho_{2}}\right)[[t]]_{s}$. To prove the surjectivity of $D$, we shall use below an approach based on Nagumo norms [9,26] and majorant series; approach which is similar to the ones developed by W. Balser and M. Loday-Richaud in [5] and by the author in [32] for some classes of linear partial differential equations. 
Definition 3.6 (Nagumo norms) Let $f \in \mathcal{O}\left(D_{\rho}\right), q \geq 0$ and $0<r \leq \rho$. Let $d_{r}(x)=r-|x|$ denote the Euclidian distance of $x \in D_{r}$ to the boundary of the disc $D_{r}$. Then, the Nagumo norm $\|f\|_{q, r}$ of $f$ is defined by

$$
\|f\|_{q, r}:=\sup _{x \in D_{r}}\left|f(x) d_{r}(x)^{q}\right| .
$$

Proposition 3.7 (Properties of Nagumo norms) Let $f, g \in \mathcal{O}\left(D_{\rho}\right)$. Let $q, q^{\prime} \geq 0$ and $0<r \leq \rho$. One has the following properties:

1. $\|\cdot\|_{q, r}$ is a norm on $\mathcal{O}\left(D_{\rho}\right)$.

2. For all $x \in D_{r},|f(x)| \leq\|f\|_{q, r} d_{r}(x)^{-q}$.

3. $\|f\|_{0, r}=\sup _{x \in D_{r}}|f(x)|$ is the usual sup-norm on $D_{r}$.

4. $\|f g\|_{q+q^{\prime}, r} \leq\|f\|_{q, r}\|g\|_{q^{\prime}, r}$.

5. $\left\|\partial_{x} f\right\|_{q+1, r} \leq e(q+1)\|f\|_{q, r}$.

Note that the same index $r$ occurs on both sides of inequalities 4 and 5 . In particular, we get estimates for the product $f g$ in terms of $f$ and $g$ and for the derivative $\partial_{x} f$ in terms of $f$ without having to shrink the disc $D_{r}$.

Let us now turn to the proof of the surjectivity of $D$. Let us fix $\widetilde{f}(t, x)=$ $\sum_{j \geq 0} f_{j, *}(x) \frac{t^{j}}{j !} \in \mathcal{O}\left(D_{\rho_{2}}\right)[[t]]_{s}$ and let us write $\widetilde{u}(t, x) \in \mathcal{O}\left(D_{\rho_{2}}\right)[[t]]$ in the same form $\widetilde{u}(t, x)=\sum_{j \geq 0} u_{j, *}(x) \frac{t^{j}}{j !}$. By assumption, the coefficients $f_{j, *}(x)$ satisfy conditions

- $f_{j, *}(x) \in \mathcal{O}\left(D_{\rho_{2}}\right)$ for all $j \geq 0$,

- there exist $0<r_{2} \leq \rho_{2}, C>0$ and $K>0$ such that $\left|f_{j, *}(x)\right| \leq C K^{j} \Gamma(1+$ $(s+1) j$ ) for all $j \geq 0$ and $x \in D_{r_{2}}$.

We shall now prove that the coefficients $u_{j, *}(x)$ satisfy similar conditions. Calculations below are analogous to those detailed in $[5,32]$, but are much more complicated because of the many terms $\partial_{t}^{-i} \partial_{x}^{q}$.

$\triangleleft$ From now on, we denote by $\kappa$ the maximum of the $i \in \mathcal{K}$ (hence, $1 \leq i \leq \kappa$ for all $i \in \mathcal{K}$ ). We also denote by $p$ the positive integer defined by

$$
p:=\left\{\begin{array}{ll}
\kappa & \text { if } \mathcal{I}_{\mathcal{K}}=\emptyset \\
p_{i_{0}} & \text { if } \mathcal{I}_{\mathcal{K}} \neq \emptyset
\end{array} .\right.
$$

From identities (1.2), it results relations

$$
\frac{u_{j, *}(x)}{\Gamma(1+(s+1) j)}=\frac{f_{j, *}(x)}{\Gamma(1+(s+1) j)}+\sum_{i \in \mathcal{K}} \sum_{q=0}^{p_{i}} \sum_{m=0}^{j-i}\left(\begin{array}{c}
j \\
m
\end{array}\right) a_{m, *}^{(i, q)}(x) \frac{\partial_{x}^{q} u_{j-m-i, *}(x)}{\Gamma(1+(s+1) j)}
$$


for all $j \geq 0$ (as before, we use the classical convention that the third sum is 0 if $j<i)$. Applying then the Nagumo norm of indices $\left(p j, r_{2}\right)$, we deduce from property 4 of proposition 3.7 that

$$
\begin{aligned}
\frac{\left\|u_{j, *}(x)\right\|_{p j, r_{2}}}{\Gamma(1+(s+1) j)} & \leq \frac{\left\|f_{j, *}(x)\right\|_{p j, r_{2}}}{\Gamma(1+(s+1) j)}+ \\
\sum_{i \in \mathcal{K}} \sum_{q=0}^{p_{i}} \sum_{m=0}^{j-i} & \left(\begin{array}{c}
j \\
m
\end{array}\right)\left\|a_{m, *}^{(i, q)}(x)\right\|_{p(m+i)-q, r_{2}} \frac{\left\|\partial_{x}^{q} u_{j-m-i, *}(x)\right\|_{p(j-m-i)+q, r_{2}}}{\Gamma(1+(s+1) j)}
\end{aligned}
$$

and from property 5 of proposition 3.7 that

$$
\begin{aligned}
& \frac{\left\|u_{j, *}(x)\right\|_{p j, r_{2}}}{\Gamma(1+(s+1) j)} \leq \frac{\left\|f_{j, *}(x)\right\|_{p j, r_{2}}}{\Gamma(1+(s+1) j)}+ \\
& \quad \sum_{i \in \mathcal{K}} \sum_{q=0}^{p_{i}} \sum_{m=0}^{j-i} e^{q} A_{i, q, m} \frac{\left\|a_{m, *}^{(i, q)}(x)\right\|_{p(m+i)-q, r_{2}}}{m !}\left\|u_{j-m-i, *}(x)\right\|_{p(j-m-i), r_{2}}
\end{aligned}
$$

where

$$
A_{i, q, m}:=\frac{\left(\prod_{\ell=0}^{m-1}(j-\ell)\right)\left(\prod_{\ell^{\prime}=0}^{q-1}\left(p(j-m-i)+q-\ell^{\prime}\right)\right)}{\Gamma(1+(s+1) j)}
$$

with the convention that the first product is 1 when $m=0$ and the second product is 1 when $q=0$. Note that the norms $\left\|a_{m, *}^{(i, q)}(x)\right\|_{p(m+i)-q, r_{2}}$ are welldefined for all $i, q$ and $m$. Indeed, in the case $\mathcal{I}_{\mathcal{K}}=\emptyset$, conditions $\kappa \geq i \geq 1$ and $i \geq p_{i}$ imply

$$
p(m+i)-q \geq p i-q=\kappa i-q \geq \kappa i-p_{i} \geq i(\kappa-1) \geq 0
$$

and, in the opposite case $\mathcal{I}_{\mathcal{K}} \neq \emptyset$, relations (3.1) and condition $i_{0} \geq 1$ imply

$$
p(m+i)-q \geq p i-q=p_{i_{0}} i-q \geq p_{i} i_{0}-q \geq p_{i} i_{0}-p_{i}=p_{i}\left(i_{0}-1\right) \geq 0 .
$$

Following technical lemmas allow to bound the $A_{i, q, m}$ 's.

Lemma 3.8 Let $i \in \mathcal{K}$ and $j \geq i$. Then, for all $m \in\{0, \ldots, j-i\}$,

$$
\frac{\prod_{\ell=0}^{m-1}(j-\ell)}{\Gamma(1+(s+1) j)} \leq \frac{1}{\Gamma(1+(s+1)(j-m))} .
$$

Proof. Lemma 3.8 is clear for $m=0$. For $m \geq 1$, we deduce from identity

$$
\Gamma(1+(s+1) j)=\Gamma(1+(s+1) j-m) \prod_{\ell=0}^{m-1}((s+1) j-\ell)
$$

the following

$$
\frac{\prod_{\ell=0}^{m-1}(j-\ell)}{\Gamma(1+(s+1) j)}=\frac{\prod_{\ell=0}^{m-1} \frac{j-\ell}{(s+1) j-\ell}}{\Gamma(1+(s+1) j-m)} \leq \frac{1}{\Gamma(1+(s+1) j-m)} .
$$


Lemma 3.8 follows then from inequalities

$$
1+(s+1) j-m \geq 1+(s+1)(j-m) \geq 1+(s+1) i \geq 2
$$

(indeed, $i \in \mathcal{K} \Rightarrow i \geq 1$ ) and from the increase of the Gamma function on $[2,+\infty[$.

Lemma 3.9 Let $i \in \mathcal{K}, q \in\left\{0, \ldots, p_{i}\right\}$ and $j \geq i$. Then, for all $m \in\{0, \ldots, j-i\}$,

$$
\frac{\prod_{\ell^{\prime}=0}^{q-1}\left(p(j-m-i)+q-\ell^{\prime}\right)}{\Gamma(1+(s+1)(j-m))} \leq \frac{\kappa^{q}}{\Gamma(1+(s+1)(j-m-i))} .
$$

Proof. • Let us first assume $\mathcal{I}_{\mathcal{K}}=\emptyset$ (hence, $p=\kappa$ and $s=0$ ). Since $q \leq p_{i} \leq$ $i \leq \kappa$, identities

$$
\prod_{\ell^{\prime}=0}^{q-1}\left(p(j-m-i)+q-\ell^{\prime}\right)=\kappa^{q} \prod_{\ell^{\prime}=0}^{q-1}\left(j-m-i+\frac{q-\ell^{\prime}}{\kappa}\right)
$$

and

$$
\Gamma(1+(s+1)(j-m))=\Gamma(1+j-m)=\Gamma(1+j-m-i) \prod_{\ell^{\prime}=0}^{i-1}\left(j-m-\ell^{\prime}\right)
$$

imply relation

$$
\frac{\prod_{\ell^{\prime}=0}^{q-1}\left(p(j-m-i)+q-\ell^{\prime}\right)}{\Gamma(1+(s+1)(j-m))} \leq \frac{\kappa^{q}}{\Gamma(1+j-m-i)} \frac{\prod_{\ell^{\prime}=0}^{q-1} \frac{j-m-i+\frac{q-\ell^{\prime}}{\kappa}}{j-m-\ell^{\prime}}}{\prod_{\ell^{\prime}=q}^{i-1}\left(j-m-\ell^{\prime}\right)}
$$

with, when the products make sense,

$$
\frac{j-m-i+\frac{q-\ell^{\prime}}{\kappa}}{j-m-\ell^{\prime}} \leq 1 \text { and } j-m-\ell^{\prime} \geq 1
$$

Note that the first inequality of (3.2) stems from inequalities

$$
-i+\frac{q-\ell^{\prime}}{\kappa}+\ell^{\prime} \leq-i+\frac{q}{\kappa}+i-1 \leq \frac{q}{\kappa}-1 \leq 0
$$

(indeed, we have $0 \leq \ell^{\prime} \leq q-1 \leq i-1$ and $q \leq \kappa$ ). As for the second inequality of (3.2), it is straightforward from inequalities $\ell^{\prime} \leq i-1$ and $m \leq j-i$. Hence, the following

$$
\frac{\prod_{\ell^{\prime}=0}^{q-1}\left(p(j-m-i)+q-\ell^{\prime}\right)}{\Gamma(1+(s+1)(j-m))} \leq \frac{\kappa^{q}}{\Gamma(1+j-m-i)}=\frac{\kappa^{q}}{\Gamma(1+(s+1)(j-m-i))}
$$


which proves lemma 3.9 for $\mathcal{I}_{\mathcal{K}}=\emptyset$.

- Let us now assume $\mathcal{I}_{\mathcal{K}} \neq \emptyset$ (hence, $p=p_{i_{0}}$ and $s=p_{i_{0}} / i_{0}-1$ ). When $m<j-i$, we proceed in a similar way as the case $\mathcal{I}_{\mathcal{K}}=\emptyset$. Let us first observe that condition $\kappa \geq i_{0}$ implies $p / \kappa \leq s+1$ and, thereby,

$$
\prod_{\ell^{\prime}=0}^{q-1}\left(p(j-m-i)+q-\ell^{\prime}\right) \leq \kappa^{q} \prod_{\ell^{\prime}=0}^{q-1}\left((s+1)(j-m-i)+\frac{q-\ell^{\prime}}{\kappa}\right) .
$$

Writing then $\Gamma(1+(s+1)(j-m))$ in the form

$$
\Gamma(1+(s+1)(j-m))=\Gamma(1+(s+1)(j-m)-q) \prod_{\ell^{\prime}=0}^{q-1}\left((s+1)(j-m)-\ell^{\prime}\right)
$$

(note that $\Gamma(1+(s+1)(j-m)-q)$ is well-defined since conditions $(m<j-i ; q \leq$ $\left.p_{i}\right)$ and relations $(3.1)$ imply $\left.1+(s+1)(j-m)-q>1+(s+1) i-p_{i} \geq 1\right)$, we get

$$
\frac{\prod_{\ell^{\prime}=0}^{q-1}\left(p(j-m-i)+q-\ell^{\prime}\right)}{\Gamma(1+(s+1)(j-m))} \leq \frac{\kappa^{q} \prod_{\ell^{\prime}=0}^{q-1} \frac{(s+1)(j-m-i)+\frac{q-\ell^{\prime}}{\kappa}}{\Gamma\left(1+(s+1)(j-m)-\ell^{\prime}\right.}}{\Gamma-m)-q)}
$$

where the product on the right-hand side is $\leq 1$. Indeed, inequalities (3.1) implying $(s+1) i \geq p_{i}$, it stems from conditions $q \leq p_{i}$ and $\kappa \geq 1$ that relations

$$
-(s+1) i+\frac{q-\ell^{\prime}}{\kappa}+\ell^{\prime} \leq\left(p_{i}-\ell^{\prime}\right)\left(\frac{1}{\kappa}-1\right) \leq 0
$$

hold for all $\ell^{\prime}$. Lemma 3.9 follows then from inequalities

$1+(s+1)(j-m)-q \geq 1+(s+1)(j-m)-p_{i} \geq 1+(s+1)(j-m-i) \geq 1+(s+1) \geq 2$

and from the increase of the Gamma function on $[2,+\infty[$. Note that the second inequality stems again from relations (3.1) and that the third inequality stems from condition $m<j-i$.

In particular, this latter inequality shows that calculations above do not allow to prove lemma 3.9 when $m=j-i$, since it fails in this case. To get around this problem, we shall proceed as follows. Let us first recall we must prove the following

$$
\frac{\prod_{\ell^{\prime}=0}^{q-1}\left(q-\ell^{\prime}\right)}{\Gamma(1+(s+1) i)} \leq \frac{\kappa^{q}}{\Gamma(1)}=\kappa^{q} .
$$

For all $q \in\left\{0, \ldots, p_{i}\right\}$, we have

$$
\prod_{\ell^{\prime}=0}^{q-1}\left(q-\ell^{\prime}\right)=\Gamma(1+q) \leq \Gamma\left(1+p_{i}\right)
$$


On the other hand, if $p_{i}=0$, inequality $1+(s+1) i \geq 2$ implies $\Gamma(1+(s+1) i) \geq$ $\Gamma(2)=1=\Gamma\left(1+p_{i}\right)$ and, if $p_{i} \geq 1$, inequalities $1+(s+1) i \geq 1+p_{i} \geq 2$ (use again relations $(3.1))$ imply $\Gamma(1+(s+1) i) \geq \Gamma\left(1+p_{i}\right)$ too. Consequently,

$$
\frac{\prod_{\ell^{\prime}=0}^{q-1}\left(q-\ell^{\prime}\right)}{\Gamma(1+(s+1) i)} \leq \frac{\Gamma\left(1+p_{i}\right)}{\Gamma\left(1+p_{i}\right)}=1 \leq \kappa^{q} \quad(\text { since } \kappa \geq 1) .
$$

Hence, inequality (3.3). This achieves the proof.

Applying then lemmas 3.8 and 3.9, we get

$$
A_{i, q, m} \leq \frac{\kappa^{q}}{\Gamma(1+(s+1)(j-m-i))}
$$

and, thereby, the following inequalities

$$
\frac{\left\|u_{j, *}(x)\right\|_{p j, r_{2}}}{\Gamma(1+(s+1) j)} \leq g_{j}+\sum_{i \in \mathcal{K}} \sum_{m=0}^{j-i} \alpha_{i, m} \frac{\left\|u_{j-m-i, *}(x)\right\|_{p(j-m-i), r_{2}}}{\Gamma(1+(s+1)(j-m-i))}
$$

hold for all $j \geq 0$ with

$$
g_{j}:=\frac{\left\|f_{j, *}(x)\right\|_{p j, r_{2}}}{\Gamma(1+(s+1) j)} \quad \text { and } \quad \alpha_{i, m}:=\sum_{q=0}^{p_{i}}(e \kappa)^{q} \frac{\left\|a_{m, *}^{(i, q)}(x)\right\|_{p(m+i)-q, r_{2}}}{m !} .
$$

$\triangleleft$ Let us now bound the Nagumo norms $\left\|u_{j, *}(x)\right\|_{p j, r_{2}}$. To do that, we shall use a technique of majorant series. Let us consider the nonnegative numerical sequence $\left(v_{j}\right)$ defined for all $j \geq 0$ by the recurrence relations

$$
v_{j}=g_{j}+\sum_{i \in \mathcal{K}} \sum_{m=0}^{j-i} \alpha_{i, m} v_{j-m-i}
$$

where, as above, the sum is 0 when $j<i$. By construction, we have

$$
0 \leq \frac{\left\|u_{j, *}(x)\right\|_{p j, r_{2}}}{\Gamma(1+(s+1) j)} \leq v_{j} \quad \text { for all } j \geq 0 .
$$

Furthermore, the $v_{j}$ 's can be bounded as follows. By assumption on the $f_{j, *}(x)$ (see the beginning of section 3.3), we have

$$
0 \leq g_{j} \leq \frac{C K^{j} \Gamma(1+(s+1) j)}{\Gamma(1+(s+1) j)} r_{2}^{p j}=C\left(K r_{2}^{p}\right)^{j}
$$

for all $j \geq 0$ and the series $g(X):=\sum_{j \geq 0} g_{j} X^{j}$ is convergent. On the other hand, all the coefficients $a^{(i, q)}(t, x)$ belong to $\mathcal{O}\left(D_{\rho_{2}}\right)\{t\}$. Then, there exist two positive constants $C^{\prime}, K^{\prime}>0$ such that $\left|a_{m, *}^{(i, q)}(x)\right| \leq C^{\prime} K^{\prime m} m$ ! for all $i \in \mathcal{K}$, $q \in\left\{0, \ldots, p_{i}\right\}, m \geq 0$ and $x \in D_{r_{2}}$. Hence,

$$
0 \leq \alpha_{i, m} \leq \sum_{q=0}^{p_{i}} \frac{(e \kappa)^{q} C^{\prime} K^{\prime m} m !}{m !} r_{2}^{p(m+i)-q}=C_{1}^{\prime}\left(K^{\prime} r_{2}^{p}\right)^{m}
$$


with $C_{1}^{\prime}=C^{\prime} r_{2}^{p i} \sum_{q=0}^{p_{i}}\left(\frac{e \kappa}{r_{2}}\right)^{q}>0$ and, consequently, the series $A_{i}(X):=\sum_{j \geq 0} \alpha_{i, j} X^{j}$ are convergent for all $i \in \mathcal{K}$ too. In particular, these calculations show us that the series $v(X):=\sum_{j \geq 0} v_{j} X^{j}$ is also convergent. Indeed, due to the recurrence relation on the $v_{j}$ 's, the series $v(X)$ satisfies the identity

$$
\left(1-\sum_{i \in \mathcal{K}} X^{i} A_{i}(X)\right) v(X)=g(X)
$$

Therefore, there exist $C^{\prime \prime}, K^{\prime \prime}>0$ such that $v_{j} \leq C^{\prime \prime} K^{\prime \prime j}$ for all $j \geq 0$. Hence, the following inequalities

$$
\left\|u_{j, *}(x)\right\|_{p j, r_{2}} \leq C^{\prime \prime} K^{\prime \prime j} \Gamma(1+(s+1) j) \quad \text { for all } j \geq 0
$$

and we are left to prove similar estimates on the sup-norm of the $u_{j, *}(x)$ 's. To this end, we proceed by shrinking the domain $D_{r_{2}}$. Let $0<r_{2}^{\prime}<r_{2}$. Then, for all $j \geq 0$ and $x \in D_{r_{2}^{\prime}}$, we have

$$
\left|u_{j, *}(x)\right|=\left|u_{j, *}(x) d_{r_{2}}(x)^{p j} \frac{1}{d_{r_{2}}(x)^{p j}}\right| \leq \frac{1}{\left(r_{2}-r_{2}^{\prime}\right)^{p j}}\left|u_{j, *}(x) d_{r_{2}}(x)^{p j}\right|
$$

and, consequently,

$$
\sup _{x \in D_{r_{2}^{\prime}}}\left|u_{j, *}(x)\right| \leq \frac{\left\|u_{j, *}(x)\right\|_{p j, r_{2}}}{\left(r_{2}-r_{2}^{\prime}\right)^{p j}} \leq C^{\prime \prime}\left(\frac{K^{\prime \prime}}{\left(r_{2}-r_{2}^{\prime}\right)^{p}}\right)^{j} \Gamma(1+(s+1) j) .
$$

This achieves the proof of the main theorem 3.4.

Remark 3.10 When $\mathcal{I}_{\mathcal{K}}=\emptyset$, calculations above show that just the condition $p_{i} \leq i$ is required. In particular, condition $\left(C_{4}\right)$ on the $a^{\left(i, p_{i}\right)}(0, x)$ 's may fail and, as we previously said, point 1 of corollary 3.5 is actually valid for any integro-differential equation.

\section{Summability of $\widetilde{u}(t, x)$}

In previous section 3 , we have shown that the formal solution $\widetilde{u}(t, x)$ and the inhomogeneity $\tilde{f}(t, x)$ of equation (1.1) are together $s$-Gevrey for a convenient $s \geq 0$ (see theorem 3.4). In particular, this has allowed us to display in the case $\mathcal{I}_{\mathcal{K}}=\emptyset$ a necessary and sufficient condition under which $\widetilde{u}(t, x)$ is convergent (see corollary 3.5 ).

In the present section, we consider the opposite case $\mathcal{I}_{\mathcal{K}} \neq \emptyset$. Moreover, we assume from now on that equation (1.1) satisfies besides the following additional two conditions

$\left(C_{5}\right) i_{0}=\kappa$ the maximum of the $i \in \mathcal{K}$,

$\left(C_{6}\right) a^{\left(\kappa, p_{\kappa}\right)}(0,0) \neq 0$. 
Note that condition $\left(C_{5}\right)$ implies, on one hand, that the slope $k=\frac{i_{0}}{p_{i_{0}}-i_{0}}=$ $\frac{\kappa}{p_{\kappa}-\kappa}$ is actually the unique positive slope of the Newton polygon $N_{t}(D)$ of operator $D$ (see section 3.1) and, on the other hand, that inequalities (3.1) become

$$
\frac{p_{\kappa}}{\kappa} \geq \frac{p_{i}}{i} \quad \text { for all } i \in \mathcal{K} .
$$

Under these conditions, we propose here below to prove a necessary and sufficient condition under which $\widetilde{u}(t, x)$ is $k$-summable in a given direction $\arg (t)=\theta$.

Remark 4.1 When condition $\left(C_{5}\right)$ fails, the Newton polygon $N_{t}(D)$ of $D$ may have several positive slopes. Then, as in the theory of linear ordinary differential equations (see for instance $[2,4,8,14,15,21,22]$ etc.), the notion of $k$-summability ceases generally to be sufficient and must be replaced by the notion of multisummability. This will be investigated in further articles.

\subsection{Main result}

Before stating the main result of this section, let us start by a preliminary remark on $\widetilde{u}(t, x)$. Writing the coefficients $a^{(i, q)}(t, x)$ of $D$ on the form $a^{(i, q)}(t, x)=$ $\sum_{n \geq 0} a_{*, n}^{(i, q)}(t) \frac{x^{n}}{n !}$ with $a_{*, n}^{(i, q)}(t) \in \mathcal{O}\left(D_{\rho_{1}}\right)$, an identification of the powers in $x$ in equation

$$
D\left(\sum_{n \geq 0} \widetilde{u}_{*, n}(t) \frac{x^{n}}{n !}\right)=\sum_{n \geq 0} \widetilde{f}_{*, n}(t) \frac{x^{n}}{n !}
$$

provides for all $n \geq 0$ the recurrence relations

$$
\begin{array}{r}
a_{*, 0}^{\left(\kappa, p_{\kappa}\right)}(t) \partial_{t}^{-\kappa} \widetilde{u}_{*, n+p_{\kappa}}=\widetilde{u}_{*, n}-\widetilde{f}_{*, n}-\sum_{m=1}^{n}\left(\begin{array}{c}
n \\
m
\end{array}\right) a_{*, m}^{\left(\kappa, p_{\kappa}\right)}(t) \partial_{t}^{-\kappa} \widetilde{u}_{*, n-m+p_{\kappa}} \\
-\sum_{i \in \mathcal{K}} \sum_{q \in Q_{i}} \sum_{m=0}^{n}\left(\begin{array}{c}
n \\
m
\end{array}\right) a_{*, m}^{(i, q)}(t) \partial_{t}^{-i} \widetilde{u}_{*, n-m+q}
\end{array}
$$

where the $Q_{i}$ 's are defined by

$$
Q_{i}=\left\{\begin{array}{ll}
\left\{0, \ldots, p_{i}\right\} & \text { if } i<\kappa \\
\left\{0, \ldots, p_{\kappa}-1\right\} & \text { if } i=\kappa
\end{array} .\right.
$$

In particular, these relations tell us that each $\widetilde{u}_{*, \ell}(t)$ (hence, $\widetilde{u}(t, x)$ too) is uniquely determined from $\widetilde{f}(t, x)$ and from the $\widetilde{u}_{*, n}(t)$ with $n=0, \ldots, p_{\kappa}-1$. Indeed, condition $\left(C_{6}\right)$ implying $a_{*, 0}^{\left(\kappa, p_{\kappa}\right)}(0) \neq 0$, the quotient $1 / a_{*, 0}^{\left(\kappa, p_{\kappa}\right)}(t)$ is welldefined in $\mathbb{C}[[t]]$.

More precisely, we have the following main result.

Theorem 4.2 Let us assume that equation (1.1) satisfies $\mathcal{I}_{\mathcal{K}} \neq \emptyset$ and conditions $\left(C_{1}\right)-\left(C_{6}\right)$. Let $s=p_{\kappa} / \kappa-1, k=1 / s$ and $\arg (t)=\theta \in \mathbb{R} / 2 \pi \mathbb{Z}$ a direction issuing from 0 . Then, 
1. The unique formal series solution $\widetilde{u}(t, x) \in \mathcal{O}\left(D_{\rho_{2}}\right)[[t]]$ of equation (1.1) is $k$-summable in direction $\theta$ if and only if the inhomogeneity $\widetilde{f}(t, x)$ and the coefficients $\widetilde{u}_{*, n}(t) \in \mathbb{C}[[t]]$ for $n=0, \ldots, p_{\kappa}-1$ are $k$-summable in direction $\theta$.

2. Moreover, the $k$-sum $u(t, x)$ in direction $\theta$, if any exists, satisfies equation (1.1) in which $\widetilde{f}(t, x)$ is replaced by its $k$-sum $f(t, x)$ in direction $\theta$.

Note that the necessary condition of point 1 is straigthforward from proposition 2.4 (indeed, $\widetilde{u}_{*, n}(t)=\partial_{x}^{n} \widetilde{u}(t, x)_{\mid x=0}$ and $\left.\widetilde{f}=D \widetilde{u}\right)$ and that point 2 stems obvious from corollary 2.5. Thereby, we are left to prove the sufficient condition of point 1 . To do that, we shall proceed through a fixed point method similar to the ones already used by W. Balser and M. Loday-Richaud in [5] and by the author in [32].

\subsection{Proof of theorem 4.2}

As we said just above, it remains to prove the sufficient condition of point 1 .

Let us write $\widetilde{u}(t, x)$ on the form

$$
\widetilde{u}(t, x)=\sum_{n=0}^{p_{\kappa}-1} \widetilde{u}_{*, n}(t) \frac{x^{n}}{n !}+\partial_{x}^{-p_{\kappa}} \widetilde{v}(t, x)
$$

with $\widetilde{v}(t, x) \in \mathcal{O}\left(D_{\rho_{2}}\right)[[t]]$ and let us set $\widetilde{w}:=\partial_{t}^{-\kappa} \widetilde{v}$. Then, since condition $\left(C_{6}\right)$ implies that $1 / a^{\left(\kappa, p_{\kappa}\right)}(t, x)$ is well-defined and holomorphic in a neighborhood of $(0,0) \in \mathbb{C}^{2}$ and since $\partial_{t}^{\kappa-i} \partial_{t}^{-\kappa}=\partial_{t}^{-i}$ for all $i \in \mathcal{K}$ (indeed, we have $i \leq \kappa$ by definition of $\kappa$ ), equation (1.1) becomes

$$
\Delta \widetilde{w}=\widetilde{g}(t, x)
$$

with

$$
\begin{aligned}
& \text { - } \Delta=1-b^{\left(\kappa, p_{\kappa}\right)}(t, x) \partial_{x}^{-p_{\kappa}} \partial_{t}^{\kappa}+\sum_{i \in \mathcal{K}} \sum_{q \in Q_{i}} b^{(i, q)}(t, x) \partial_{x}^{q-p_{\kappa}} \partial_{t}^{\kappa-i}, \\
& \text { - } \widetilde{g}=\frac{1}{a^{\left(\kappa, p_{\kappa}\right)}}\left(\sum_{n=0}^{p_{\kappa}-1} \widetilde{u}_{*, n}(t) \frac{x^{n}}{n !}-\sum_{i \in \mathcal{K}} \sum_{q \in Q_{i}} \sum_{n=0}^{p_{\kappa}-1-q} a^{(i, q)} \widetilde{u}_{*, n+q}(t) \frac{x^{n}}{n !}-\tilde{f}\right) .
\end{aligned}
$$

The $Q_{i}$ 's are the sets already introduced in $(4.2)$ and the $b^{(i, q)}$ 's are the holomorphic functions defined by

$$
b^{(i, q)}=\left\{\begin{array}{ll}
\frac{1}{a^{\left(\kappa, p_{\kappa}\right)}} & \text { if }(i, q)=\left(\kappa, p_{\kappa}\right) \\
\frac{a^{(i, q)}}{a^{\left(\kappa, p_{\kappa}\right)}} & \text { if }(i, q) \neq\left(\kappa, p_{\kappa}\right)
\end{array} .\right.
$$

We denote below by $D_{\rho_{1}^{\prime}} \times D_{\rho_{2}^{\prime}}$, with $\rho_{1}^{\prime}, \rho_{2}^{\prime}>0$, the common domain of $(0,0) \in$ $\mathbb{C}^{2}$ where all the $b^{(i, q)}$ 's are holomorphic.

Let us now assume $\widetilde{f}(t, x)$ and the $\widetilde{u}_{*, n}(t)$ 's $k$-summable in a given direction $\theta$. Then, $\widetilde{g}(t, x)$ is $k$-summable in direction $\theta$ (see proposition 2.4) and calculations above tell us it suffices to prove that it is the same for $\widetilde{w}(t, x)$. To this 
end, we shall proceed similarly as $[5,32]$ through a fixed point method.

Let us set $\widetilde{w}(t, x)=\sum_{m>0} \widetilde{w}_{m}(t, x)$ and let us consider the solution of equation (4.3), where the $\widetilde{w}_{m}(t, x)^{\prime}$ 's belong to $\mathcal{O}\left(D_{\rho}\right)[[t]]$ for a suitable common $\rho>0$ and are recursively determined, for all $m \geq 0$, by the relations

$$
\left\{\begin{array}{l}
\widetilde{w}_{0}=\widetilde{g} \\
\widetilde{w}_{m+1}=b^{\left(\kappa, p_{\kappa}\right)}(t, x) \partial_{x}^{-p_{\kappa}} \partial_{t}^{\kappa} \widetilde{w}_{m}-\sum_{i \in \mathcal{K}} \sum_{q \in Q_{i}} b^{(i, q)}(t, x) \partial_{x}^{q-p_{\kappa}} \partial_{t}^{\kappa-i} \widetilde{w}_{m}
\end{array}\right.
$$

Note that, for all $m \geq 0$, the formal series $\widetilde{w}_{m}(t, x)$ are of order $O\left(x^{m}\right)$ in $x$ and, consequently, the series $\widetilde{w}(t, x)$ itself makes sense as a formal series in $t$ and $x$.

Let us now denote by $w_{0}(t, x)$ the $k$-sum of $\widetilde{w}_{0}=\widetilde{g}$ in direction $\theta$ and, for all $m \geq 0$, let $w_{m}(t, x)$ be determined as the solution of system (4.4) in which all the $\widetilde{w}_{m}$ are replaced by $w_{m}$. By construction, all the $w_{m}(t, x)$ are defined and holomorphic on a common domain $\Sigma_{\theta,>\pi s} \times D_{\rho_{2}^{\prime \prime}}$, where the radius $\rho_{1}^{\prime \prime}$ of $\Sigma_{\theta,>\pi s}$ and the radius $\rho_{2}^{\prime \prime}$ of $D_{\rho_{2}^{\prime \prime}}$ can always be chosen so that $0<\rho_{1}^{\prime \prime}<\rho_{1}^{\prime}$ and $0<\rho_{2}^{\prime \prime}<\min \left(\rho_{2}, \rho_{2}^{\prime}\right)$. To end the proof, it remains to prove that the series $\sum_{m \geq 0} w_{m}(t, x)$ is convergent and that its sum $w(t, x)$ is the $k$-sum of $\widetilde{w}(t, x)$ in direction $\theta$.

According to definition 2.3, the $k$-summability of $\widetilde{w}_{0}$ implies that there exists $0<r_{2}<\rho_{2}^{\prime \prime}$ such that, for any proper subsector $\Sigma \Subset \Sigma_{\theta,>\pi s}$, there exist constants $C>0$ and $K \geq 1$ such that, for all $\ell \geq 0$ and all $(t, x) \in \Sigma \times D_{r_{2}}$, the function $w_{0}$ satisfies the inequalities

$$
\left|\partial_{t}^{\ell} w_{0}(t, x)\right| \leq C K^{\ell} \Gamma(1+(s+1) \ell) .
$$

Let us now fix a proper subsector $\Sigma \Subset \Sigma_{\theta,>\pi s}$ and let us denote by $r_{1}$ its radius. Note that inequalities (4.5) still hold with the same constants $C$ and $K$ for any $0<r_{2}^{\prime}<r_{2}$. In particular, we can always assume in the sequel that $r_{2}<1$.

Proposition 4.3 below provides us some estimates on the derivatives $\partial_{t}^{\ell} w_{m}$ of $w_{m}$. Before stating it, let us first begin by given some estimates on the holomorphic functions $\partial_{t}^{\ell} b^{(i, q)}$. Let

$$
B:=\max _{(i, q)}\left(\max _{(t, x) \in \bar{D}_{\rho_{1}^{\prime \prime}} \times \bar{D}_{\rho_{2}^{\prime \prime}}}\left|b^{(i, q)}(t, x)\right|\right),
$$

where $\bar{D}_{\rho}$ denotes the closed disc with center 0 and radius $\rho>0$. Note that $B$ is well-defined since all the $b^{(i, q)}$ are holomorphic on $D_{\rho_{1}^{\prime}} \times D_{\rho_{2}^{\prime}}$ and $0<\rho_{j}^{\prime \prime}<\rho_{j}^{\prime}$ for $j=1,2$. Then, the Cauchy integral formula

$$
\partial_{t}^{\ell} b^{(i, q)}(t, x)=\frac{\ell !}{(2 i \pi)^{2}} \int \begin{aligned}
& \left|t^{\prime}-t\right|=\rho_{1}^{\prime \prime}-r_{1} \\
& \left|x^{\prime}-x\right|=\rho_{2}^{\prime \prime}-r_{2}
\end{aligned} \frac{b^{(i, q)}\left(t^{\prime}, x^{\prime}\right)}{\left(t^{\prime}-t\right)^{\ell+1}\left(x^{\prime}-x\right)} d t^{\prime} d x^{\prime}
$$

implies inequalities

$$
\left|\partial_{t}^{\ell} b^{(i, q)}(t, x)\right| \leq \ell ! B\left(\frac{1}{\rho_{1}^{\prime \prime}-r_{1}}\right)^{\ell}
$$


for all $\ell \geq 0$ and $(t, x) \in \Sigma \times D_{r_{2}}$. In particular, these estimates only depend on the radius $r_{1}$ of $\Sigma$ and not on $r_{2}$. Thereby, the constant $K$ being chosen $\geq 1 /\left(\rho_{1}^{\prime \prime}-r_{1}\right)$, we get

$$
\left|\partial_{t}^{\ell} b^{(i, q)}(t, x)\right| \leq \ell ! B K^{\ell}
$$

for all $(i, q), \ell \geq 0$ and $(t, x) \in \Sigma \times D_{r_{2}}$.

Proposition 4.3 Let $B^{\prime}:=(\kappa+1)^{2} B$ and $\left(P_{m}(x)\right)$ the sequence of polynomials in $\mathbb{R}^{+}[x]$ recursively determined by

$$
\left\{\begin{array}{l}
P_{0}(x)=1 \\
P_{m+1}(x)=\left(\partial_{x}^{-p_{\kappa}}+\sum_{i \in \mathcal{K}^{\prime}} \sum_{q \in Q_{i}^{\prime}} \frac{\left(m p_{\kappa}\right) !}{\left(m p_{\kappa}+p_{i}\right) !} \partial_{x}^{-q}\right) P_{m}(x) \quad \text { for } m \geq 0
\end{array}\right.
$$

with $\mathcal{K}^{\prime}:=\left\{i \in \mathcal{K} ; p_{i} \geq 1\right\}$ and $Q_{i}^{\prime}:=\left\{\max \left(p_{\kappa}-p_{i}, 1\right), \ldots, p_{\kappa}-1\right\}$. Then, the following inequalities

$$
\left|\partial_{t}^{\ell} w_{m}(t, x)\right| \leq C B^{\prime m} K^{\kappa m+\ell} \Gamma(1+(s+1)(\kappa m+\ell)) P_{m}(|x|)
$$

hold for all $m, \ell \geq 0$ and all $(t, x) \in \Sigma \times D_{r_{2}}$.

Note that the set $\mathcal{K}^{\prime}$ is never empty since $p_{\kappa}>\kappa \geq 1$ implies $\kappa \in \mathcal{K}^{\prime}$. The following proof of proposition 4.3 proceeds by recursion on $m \geq 0$.

Proof. The case $m=0$ is straightaway from inequalities (4.5). Let us now suppose that inequalities (4.7) hold for a certain $m \geq 0$. Then, according to relations (4.4), we deduce from Leibniz formula and from inequalities (4.6) and $K \geq 1$ that, for all $\ell \geq 0$ and $(t, x) \in \Sigma \times D_{r_{2}}$,

$$
\left|\partial_{t}^{\ell} w_{m+1}(t, x)\right| \leq C B B^{\prime m} K^{\kappa(m+1)+\ell} \sum_{i \in \mathcal{K} \cup\{0\}}\left(S_{\ell, i} \sum_{q \in Q_{i}}\left(\partial_{x}^{q-p_{\kappa}} P_{m}\right)(|x|)\right),
$$

where we set $Q_{0}:=\{0\}$ and where $S_{\ell, i}$ is the sum defined by

$$
S_{\ell, i}:=\sum_{j=0}^{\ell} \frac{\ell !}{j !} \Gamma(1+(s+1)(\kappa m+\kappa+j-i)) .
$$

This latter can be bounded as follows by applying successively technical lemmas $4.4,4.5$ and 4.6 below.

$$
\begin{aligned}
S_{\ell, i} \leq & \sum_{j=0}^{\ell} \Gamma(1+(s+1)(\kappa m+\kappa+j-i)+\ell-j) \\
& =\Gamma(1+(s+1)(\kappa m+\kappa+\ell-i)) \times \\
& \sum_{j=0}^{\ell} \frac{\Gamma(1+(s+1)(\kappa m+\kappa+j-i)+\ell-j)}{\Gamma(1+(s+1)(\kappa m+\kappa+\ell-i))} \\
\leq & (\kappa+1) \Gamma(1+(s+1)(\kappa m+\kappa+\ell-i)) \\
\leq & (\kappa+1) \frac{\left(m p_{\kappa}\right) !}{\left(m p_{\kappa}+p_{i}\right) !} \Gamma(1+(s+1)(\kappa(m+1)+\ell))
\end{aligned}
$$


with the convention that $p_{0}=0$. This leads then us to the following

$$
\begin{array}{r}
\left|\partial_{t}^{\ell} w_{m+1}(t, x)\right| \leq(\kappa+1) C B B^{\prime m} K^{\kappa(m+1)+\ell} \Gamma(1+(s+1)(\kappa(m+1)+\ell)) \\
\times \sum_{i \in \mathcal{K} \cup\{0\}} \sum_{q \in Q_{i}} \frac{\left(m p_{\kappa}\right) !}{\left(m p_{\kappa}+p_{i}\right) !}\left(\partial_{x}^{q-p_{\kappa}} P_{m}\right)(|x|)
\end{array}
$$

and inequalities (4.7) follow by observing that the double-sum of the right-hand side satisfies

$$
\begin{aligned}
\sum_{i \in \mathcal{K} \cup\{0\}} \sum_{q \in Q_{i}} \frac{\left(m p_{\kappa}\right) !}{\left(m p_{\kappa}+p_{i}\right) !}\left(\partial_{x}^{q-p_{\kappa}} P_{m}\right)(|x|) & \leq(\kappa+1)\left(\partial_{x}^{-p_{\kappa}} P_{m}\right)(|x|) \\
& +\sum_{i \in \mathcal{K}^{\prime}} \sum_{q \in Q_{i}^{\prime}} \frac{\left(m p_{\kappa}\right) !}{\left(m p_{\kappa}+p_{i}\right) !}\left(\partial_{x}^{-q} P_{m}\right)(|x|)
\end{aligned}
$$

hence,

$$
\sum_{i \in \mathcal{K} \cup\{0\}} \sum_{q \in Q_{i}} \frac{\left(m p_{\kappa}\right) !}{\left(m p_{\kappa}+p_{i}\right) !}\left(\partial_{x}^{q-p_{\kappa}} P_{m}\right)(|x|) \leq(\kappa+1) P_{m+1}(|x|) .
$$

Indeed, $\left(m p_{\kappa}\right) ! /\left(m p_{\kappa}+p_{i}\right) ! \leq 1$ for all $i, \mathcal{K} \subset\{1, \ldots, \kappa\}$ and the coefficients of polynomial $P_{m}$ are positive. This ends the proof of proposition 4.3.

Lemma 4.4 Let $i \in \mathcal{K} \cup\{0\}$. Then, for all $\ell \geq 0, j \in\{0, \ldots, \ell\}$ and $m \geq 0$,

$$
\frac{\ell !}{j !} \Gamma(1+(s+1)(\kappa m+\kappa+j-i)) \leq \Gamma(1+(s+1)(\kappa m+\kappa+j-i)+\ell-j) .
$$

Proof. Lemma 4.4 is clear for $j=\ell$. Let us now assume $j<\ell$ and let us write $\ell ! / j$ ! on the form

$$
\frac{\ell !}{j !}=\prod_{n=j+1}^{\ell} n=\prod_{n=j+1}^{\ell}(j+n-j) .
$$

Then,

$$
\begin{aligned}
\frac{\ell !}{j !} & \leq \prod_{n=j+1}^{\ell}((s+1)(\kappa m+\kappa+j-i)+n-j) \\
& =\prod_{n=1}^{\ell-j}((s+1)(\kappa m+\kappa+j-i)+n)
\end{aligned}
$$

and relation

$$
\begin{aligned}
\Gamma(1+(s+1)(\kappa m+\kappa+j-i)+\ell-j) & =\Gamma(1+(s+1)(\kappa m+\kappa+j-i)) \\
& \times \prod_{n=1}^{\ell-j}((s+1)(\kappa m+\kappa+j-i)+n)
\end{aligned}
$$

completes the proof. 
Lemma 4.5 Let $i \in \mathcal{K} \cup\{0\}$. Then, for all $\ell \geq 0$ and $m \geq 0$,

$$
\sum_{j=0}^{\ell} \frac{\Gamma(1+(s+1)(\kappa m+\kappa+j-i)+\ell-j)}{\Gamma(1+(s+1)(\kappa m+\kappa+\ell-i))} \leq \kappa+1 .
$$

Proof. $\triangleleft$ Let us first suppose $\ell \leq \kappa$. When $m \neq 0$ or $i \neq \kappa$, we have, for all $j \in\{0, \ldots, \ell\}$,

$$
\begin{aligned}
& 1+(s+1)(\kappa m+\kappa+j-i)+\ell-j=1+(s+1)(\kappa m+\kappa-i)+\ell+s j \\
& \leq 1+(s+1)(\kappa m+\kappa+\ell-i)
\end{aligned}
$$

and

$$
1+(s+1)(\kappa m+\kappa+j-i)+\ell-j \geq 1+(s+1)(\kappa m+\kappa-i) \geq 2 .
$$

Hence, using the increase of the Gamma function on $[2,+\infty[$,

$$
\sum_{j=0}^{\ell} \frac{\Gamma(1+(s+1)(\kappa m+\kappa+j-i)+\ell-j)}{\Gamma(1+(s+1)(\kappa m+\kappa+\ell-i))} \leq \sum_{j=0}^{\ell} 1=\ell+1 \leq \kappa+1
$$

and so inequality (4.8). When $m=0$ and $i=\kappa$, we must prove the inequality

$$
\sum_{j=0}^{\ell} \frac{\Gamma(1+(s+1) j+\ell-j)}{\Gamma(1+(s+1) \ell)} \leq \kappa+1 .
$$

This one is clear for $\ell=0$. Otherwise, we have

$$
2 \leq 1+\ell-j \leq 1+(s+1) j+\ell-j=1+s j+\ell \leq 1+(s+1) \ell
$$

for all $j<\ell$; hence,

$$
\sum_{j=0}^{\ell} \frac{\Gamma(1+(s+1) j+\ell-j)}{\Gamma(1+(s+1) \ell)} \leq \sum_{j=0}^{\ell-1} 1+1=\ell+1 \leq \kappa+1 .
$$

$\triangleleft$ Let us now suppose $\ell>\kappa$ and let us write the sum of (4.8) on the form

$$
\sum_{j=0}^{\ell}(\ldots)=\sum_{j=0}^{\ell-\kappa}(\ldots)+\sum_{j=\ell-\kappa+1}^{\ell}(\ldots) .
$$

The second sum of the right-hand side of (4.9) is treated as in the previous case and we get

$$
\sum_{j=\ell-\kappa+1}^{\ell}(\ldots) \leq \sum_{j=\ell-\kappa+1}^{\ell} 1=\kappa .
$$

On the other hand, for $j \in\{0, \ldots, \ell-\kappa\}$, similar calculations as above lead us to the following inequalities

$$
\begin{aligned}
2 & \leq 1+\kappa \\
& \leq 1+(s+1)(\kappa m+\kappa+j-i)+\ell-j \leq 1+(s+1)(\kappa m+\kappa+\ell-i)-s \kappa .
\end{aligned}
$$


Thereby, the first sum of the right-hand side of (4.9) gives us

$$
\begin{aligned}
\sum_{j=0}^{\ell-\kappa}(\ldots) & \leq(\ell-\kappa+1) \frac{\Gamma(1+(s+1)(\kappa m+\kappa+\ell-i)-s \kappa)}{\Gamma(1+(s+1)(\kappa m+\kappa+\ell-i))} \\
& =\frac{\ell-\kappa+1}{(s+1)(\kappa m+\kappa+\ell-i)} \frac{\Gamma(1+(s+1)(\kappa m+\kappa+\ell-i)-s \kappa)}{\Gamma(1+(s+1)(\kappa m+\kappa+\ell-i)-1)} \\
& \leq \frac{\ell-\kappa+1}{(s+1)(\kappa m+\kappa+\ell-i)} .
\end{aligned}
$$

Indeed, we have $\ell>\kappa \geq 1$ and $s \kappa=p_{\kappa}-\kappa \geq 1$; hence,

$$
\begin{aligned}
2 & \leq 1+\kappa \\
& \leq 1+(s+1)(\kappa m+\kappa+\ell-i)-s \kappa \leq 1+(s+1)(\kappa m+\kappa+\ell-i)-1
\end{aligned}
$$

and, consequently,

$$
\frac{\Gamma(1+(s+1)(\kappa m+\kappa+\ell-i)-s \kappa)}{\Gamma(1+(s+1)(\kappa m+\kappa+\ell-i)-1)} \leq 1 .
$$

We then conclude by observing that

$$
\frac{\ell-\kappa+1}{(s+1)(\kappa m+\kappa+\ell-i)} \leq \frac{1}{s+1} \leq 1
$$

for all $\ell \geq 0$. This ends the proof of lemma 4.5 .

Lemma 4.6 Let $i \in \mathcal{K} \cup\{0\}$. Then, for all $\ell \geq 0$ and $m \geq 0$,

$$
\Gamma(1+(s+1)(\kappa m+\kappa+\ell-i)) \leq \frac{\left(m p_{\kappa}\right) !}{\left(m p_{\kappa}+p_{i}\right) !} \Gamma(1+(s+1)(\kappa(m+1)+\ell)),
$$

where we set $p_{0}:=0$.

Proof. Lemma 4.6 is clear for $i=0$. When $i \geq 1$, let us first observe that relations $(4.1)$, which stems from condition $\left(C_{5}\right)$, imply

$$
\begin{aligned}
1+(s+1)(\kappa(m+1)+\ell) & =1+(s+1)(\kappa m+\kappa+\ell-i)+\frac{p_{\kappa}}{\kappa} i \\
& \geq 1+(s+1)(\kappa m+\kappa+\ell-i)+p_{i} .
\end{aligned}
$$

Thereby, since

$$
1+(s+1)(\kappa m+\kappa+\ell-i)+p_{i} \geq \begin{cases}1+p_{\kappa} \geq 2 & \text { if } i=\kappa \\ 1+\kappa-i \geq 2 & \text { if } i<\kappa\end{cases}
$$

we deduce from the increase of the Gamma function on $[2,+\infty[$ that

$$
\Gamma(1+(s+1)(\kappa(m+1)+\ell)) \geq \Gamma\left(1+(s+1)(\kappa m+\kappa+\ell-i)+p_{i}\right) ;
$$

hence the inequality

$$
\begin{aligned}
\Gamma(1+(s+1)(\kappa(m+1)+\ell)) \geq \Gamma(1+ & (s+1)(\kappa m+\kappa+\ell-i)) \\
& \times \prod_{n=1}^{p_{i}}((s+1)(\kappa m+\kappa+\ell-i)+n) .
\end{aligned}
$$


Lemma 4.6 follows then from relations

$$
\begin{aligned}
\prod_{n=1}^{p_{i}}((s+1)(\kappa m+\kappa+\ell-i)+n) & \geq \prod_{n=1}^{p_{i}}((s+1) \kappa m+n) \\
& =\prod_{n=1}^{p_{i}}\left(m p_{\kappa}+n\right)=\frac{\left(m p_{\kappa}+p_{i}\right) !}{\left(m p_{\kappa}\right) !},
\end{aligned}
$$

which ends the proof.

Let us now give some estimates on the $P_{m}(x)$ 's. We have the following.

Proposition 4.7 Let $m \geq 0$. Then, $P_{m}(x)$ reads as

$$
P_{m}(x)=\frac{x^{m p_{\kappa}}}{\left(m p_{\kappa}\right) !}+\sum_{n=1}^{m} M_{n}(x),
$$

where $M_{n}(x) \in \mathbb{R}^{+}[x]$ is the polynomial with positive coefficients defined by

$$
M_{n}(x):=\sum_{\substack{\left(i_{1}, \ldots, i_{n}\right) \\
\in\left(\mathcal{K}^{\prime}\right)^{n}}} \sum_{1 \leq j_{1}<\ldots<j_{n} \leq m} \sum_{\begin{array}{c}
\left(q_{i_{1}, j_{1}}, \ldots, q_{i_{n}, j_{n}}\right) \\
\in Q_{i_{1}}^{\prime} \times \ldots \times Q_{i_{n}}
\end{array}} A_{i_{1}, \ldots, i_{n}}^{j_{1}, \ldots, j_{n}} x^{(m-n) p_{\kappa}+q_{i_{1}, j_{1}}+\ldots q_{i_{n}, j_{n}}}
$$

with

$$
A_{i_{1}, \ldots, i_{n}}^{j_{1}, \ldots, j_{n}}=\frac{1}{\left((m-n) p_{\kappa}+q_{i_{1}, j_{1}}+\ldots q_{i_{n}, j_{n}}\right) !} \prod_{\ell=1}^{n} \frac{\left(\left(j_{\ell}-1\right) p_{\kappa}\right) !}{\left(\left(j_{\ell}-1\right) p_{\kappa}+p_{i_{\ell}}\right) !} .
$$

Moreover, the coefficients $A_{i_{1}, \ldots, i_{n}}^{j_{1}, \ldots, j_{n}} \in \mathbb{R}^{+}$satisfy

$$
A_{i_{1}, \ldots, i_{n}}^{j_{1}, \ldots, j_{n}} \leq \frac{\left(2^{p_{\kappa}}\left(1+p_{\kappa}\right)^{p_{\kappa}-1}\right)^{m}}{\left(m p_{\kappa}\right) !} \text { for all } n=1, \ldots, m
$$

and the following inequality

$$
P_{m}(|x|) \leq \frac{\left(\kappa p_{\kappa} 2^{p_{\kappa}}\left(1+p_{\kappa}\right)^{p_{\kappa}-1}\right)^{m}}{\left(m p_{\kappa}\right) !}|x|^{m} .
$$

holds for all $x \in D_{r_{2}}$.

Proof. $\triangleleft$ Formula (4.10) can be proved by recursion on $m \geq 0$ and stems from the definition of the sequence $\left(P_{m}(x)\right)$ given in proposition 4.3 above. The calculations are left to the reader.

$\triangleleft$ To bound the coefficients $A_{i_{1}, \ldots, i_{n}}^{j_{1}, \ldots, j_{n}}$, we proceed as follows. Let us first denote by $a_{\ell}$ the positive integer defined by

$$
a_{\ell}:= \begin{cases}1 & \text { if } i_{\ell}=\kappa \\ p_{\kappa}-p_{i_{\ell}} & \text { if } i_{\ell} \neq \kappa\end{cases}
$$

so that $q_{i_{\ell}, j_{\ell}} \geq a_{\ell}$ for all $\ell=1, \ldots, n$. In particular, we get

$$
\begin{aligned}
\frac{1}{\left((m-n) p_{\kappa}+q_{i_{1}, j_{1}}+\ldots q_{i_{n}, j_{n}}\right) !} & \leq \frac{1}{\left((m-n) p_{\kappa}+a_{1}+\ldots+a_{n}\right) !} \\
& \leq \frac{1}{\left((m-n) p_{\kappa}\right) !} \prod_{\ell=1}^{n} B_{\ell}
\end{aligned}
$$


with

$$
B_{\ell}:=\prod_{r=1}^{a_{\ell}} \frac{1}{a_{1}+\ldots a_{\ell-1}+r} .
$$

On the other hand, we have

$$
\begin{aligned}
\prod_{\ell=1}^{n} \frac{\left(\left(j_{\ell}-1\right) p_{\kappa}\right) !}{\left(\left(j_{\ell}-1\right) p_{\kappa}+p_{i_{\ell}}\right) !} & =\prod_{\ell=1}^{n} \prod_{r=1}^{p_{i_{\ell}}} \frac{1}{\left(j_{\ell}-1\right) p_{\kappa}+r} \\
& \leq \prod_{\ell=1}^{n} \prod_{r=1}^{p_{i_{\ell}}} \frac{1}{(\ell-1) p_{\kappa}+r}=\frac{1}{\left(n p_{\kappa}\right) !} \prod_{\ell=1}^{n} B_{\ell}^{\prime}
\end{aligned}
$$

with

$$
B_{\ell}^{\prime}:=\left\{\begin{array}{ll}
1 & \text { if } i_{\ell}=\kappa \\
\prod_{r=1}^{p_{\kappa}-p_{i_{\ell}}}\left((\ell-1) p_{\kappa}+p_{i_{\ell}}+r\right) & \text { if } i_{\ell} \neq \kappa
\end{array} .\right.
$$

This brings then us to the following inequality

$$
A_{i_{1}, \ldots, i_{n}}^{j_{1}, \ldots, j_{n}} \leq \frac{1}{\left(m p_{\kappa}\right) !}\left(\begin{array}{c}
m p_{\kappa} \\
n p_{\kappa}
\end{array}\right) \prod_{\ell=1}^{n} B_{\ell} B_{\ell}^{\prime}
$$

where the product $B_{\ell} B_{\ell}^{\prime}$ satisfies

$$
B_{\ell} B_{\ell}^{\prime}=\frac{1}{a_{1}+\ldots+a_{\ell-1}+1} \leq 1=\left(1+p_{\kappa}\right)^{p_{\kappa}-p_{i_{\ell}}} \quad \text { if } i_{\ell}=\kappa
$$

and

$$
\begin{aligned}
B_{\ell} B_{\ell}^{\prime} & =\prod_{r=1}^{p_{\kappa}-p_{i_{\ell}}}\left(1+\frac{\ell p_{\kappa}-\left(p_{\kappa}-p_{i_{\ell}}\right)-a_{1}-\ldots-a_{\ell-1}}{a_{1}+\ldots+a_{\ell-1}+r}\right) \\
& \leq \prod_{r=1}^{p_{\kappa}-p_{i_{\ell}}}\left(1+\frac{\ell p_{\kappa}}{\ell}\right) \\
& =\left(1+p_{\kappa}\right)^{p_{\kappa}-p_{i_{\ell}}} \quad \text { if } i_{\ell} \neq \kappa .
\end{aligned}
$$

Indeed, we have $p_{\kappa} \geq p_{\kappa}-p_{i_{\ell}} \geq 1$ and $a_{i} \geq 1$ by definition. Hence,

$$
A_{i_{1}, \ldots, i_{n}}^{j_{1}, \ldots, j_{n}} \leq \frac{1}{\left(m p_{\kappa}\right) !}\left(\begin{array}{c}
m p_{\kappa} \\
n p_{\kappa}
\end{array}\right)\left(1+p_{\kappa}\right)^{n p_{\kappa}-p_{i_{1}}-\ldots-p_{i_{n}}}
$$

and inequality (4.11) follows from relations

$$
\left(\begin{array}{c}
m p_{\kappa} \\
n p_{\kappa}
\end{array}\right) \leq \sum_{\ell=0}^{m p_{\kappa}}\left(\begin{array}{c}
m p_{\kappa} \\
\ell
\end{array}\right)=2^{m p_{\kappa}}
$$

and

$$
0 \leq n p_{\kappa}-p_{i_{1}}-\ldots-p_{i_{n}} \leq n\left(p_{\kappa}-1\right) \leq m\left(p_{\kappa}-1\right) .
$$

Note that this latter relation stems from inequality $p_{\kappa}>\kappa \geq 1$ and from the fact that $p_{\kappa} \geq p_{i} \geq 1$ for all $i \in \mathcal{K}^{\prime}$. 
$\triangleleft$ We are left to prove inequality (4.12). This one is clear for $m=0$ since $P_{0}(x)=$ 1. For $m \geq 1$, let us first observe that the assumption $r_{2}<1$ and inequalities $p_{\kappa} \geq 1$ and $q_{i_{\ell}, j_{\ell}} \geq 1$ imply $|x|^{m p_{\kappa}} \leq|x|^{m}$ and $|x|^{(m-n) p_{\kappa}+q_{i_{1}, j_{1}}+\ldots q_{i_{n}, j_{n}}} \leq|x|^{m}$ for all $\ell=1, \ldots, n$. Then, since $2^{p_{\kappa}}\left(1+p_{\kappa}\right)^{p_{\kappa}-1} \geq 1$, we get, for all $x \in D_{r_{2}}$,

$$
P_{m}(|x|) \leq \frac{\left(2^{p_{\kappa}}\left(1+p_{\kappa}\right)^{p_{\kappa}-1}\right)^{m}}{\left(m p_{\kappa}\right) !} b_{m}|x|^{m}
$$

with

$$
\begin{aligned}
b_{m} & :=1+\sum_{n=1}^{m} \sum_{\substack{\left(i_{1}, \ldots, i_{n}\right) \\
\in\left(\mathcal{K}^{\prime}\right)^{n}}} \sum_{\substack { 1 \leq j_{1}<\ldots<j_{n} \leq m \\
\begin{subarray}{c}{\left(q_{i_{1}, j_{1}}, \ldots, q_{i_{n}, j_{n}}\right) \\
\in Q_{i_{1}}^{\prime} \times \ldots \times Q_{i_{n}}^{\prime}{ 1 \leq j _ { 1 } < \ldots < j _ { n } \leq m \\
\begin{subarray} { c } { ( q _ { i _ { 1 } , j _ { 1 } } , \ldots , q _ { i _ { n } , j _ { n } } ) \\
\in Q _ { i _ { 1 } } ^ { \prime } \times \ldots \times Q _ { i _ { n } } ^ { \prime } } }\end{subarray}} 1 \\
& \leq 1+\sum_{n=1}^{m}\left(\begin{array}{c}
m \\
n
\end{array}\right)\left(\kappa\left(p_{\kappa}-1\right)\right)^{n} \\
& =\left(1+\kappa\left(p_{\kappa}-1\right)\right)^{m} \leq\left(\kappa p_{\kappa}\right)^{m} .
\end{aligned}
$$

Indeed, we have $\mathcal{K}^{\prime} \subset \mathcal{K} \subset\{1, \ldots, \kappa\}$ and $Q_{i_{\ell}}^{\prime} \subset\left\{1, \ldots, p_{\kappa}-1\right\}$ for all $\ell=1, \ldots, n$. This proves inequality (4.12) and completes thereby the proof of proposition 4.7 .

Let $B^{\prime \prime}:=B^{\prime} K^{\kappa} \kappa p_{\kappa} 2^{p_{\kappa}}\left(1+p_{\kappa}\right)^{p_{\kappa}-1}$. Then, we deduce from propositions 4.3 and 4.7 that, for all $\ell \geq 0$ and $(t, x) \in \Sigma \times D_{r_{2}}$,

$$
\sum_{m \geq 0}\left|\partial_{t}^{\ell} w_{m}(t, x)\right| \leq C K^{\ell} \Gamma(1+(s+1) \ell) \sum_{m \geq 0} A_{m, \ell}(x)
$$

with

$$
A_{m, \ell}(x)=\frac{\Gamma(1+(s+1)(\kappa m+\ell))}{\Gamma(1+(s+1) \ell)} \frac{\left(B^{\prime \prime}|x|\right)^{m}}{\left(m p_{\kappa}\right) !} .
$$

Let us now observe that inequality $s+1 \leq p_{\kappa}$ implies

$$
\begin{aligned}
\Gamma(1+(s+1)(\kappa m+\ell)) & =\Gamma\left(1+(s+1) \ell+m p_{\kappa}\right) \\
& =\Gamma(1+(s+1) \ell) \prod_{j=1}^{m p_{\kappa}}((s+1) \ell+j) \\
& \leq \Gamma(1+(s+1) \ell) \prod_{j=1}^{m p_{\kappa}}\left(\ell p_{\kappa}+j\right) \\
& =\Gamma(1+(s+1) \ell) \frac{\left(\ell p_{\kappa}+m p_{\kappa}\right) !}{\left(\ell p_{\kappa}\right) !}
\end{aligned}
$$

and, thereby,

$$
\frac{\Gamma(1+(s+1)(\kappa m+\ell))}{\left(m p_{\kappa}\right) ! \Gamma(1+(s+1) \ell)} \leq\left(\begin{array}{c}
\ell p_{\kappa}+m p_{\kappa} \\
m p_{\kappa}
\end{array}\right) \leq \sum_{j=0}^{\ell p_{\kappa}+m p_{\kappa}}\left(\begin{array}{c}
\ell p_{\kappa}+m p_{\kappa} \\
j
\end{array}\right)=2^{\ell p_{\kappa}+m p_{\kappa}}
$$

Consequently,

$$
\sum_{m \geq 0}\left|\partial_{t}^{\ell} w_{m}(t, x)\right| \leq C\left(2^{p_{\kappa}} K\right)^{\ell} \Gamma(1+(s+1) \ell) \sum_{m \geq 0}\left(2^{p_{\kappa}} B^{\prime \prime}|x|\right)^{m}
$$


for all $\ell \geq 0$ and $(t, x) \in \Sigma \times D_{r_{2}}$. Let us now choose $0<r<\min \left(r_{2}, 2^{-p_{\kappa}} / B^{\prime \prime}\right)$ and let us denote $C^{\prime}:=C \sum_{m \geq 0}\left(2^{p_{\kappa}} B^{\prime \prime} r\right)^{m} \in \mathbb{R}^{+}$and $K^{\prime}:=2^{p_{\kappa}} K$. Then, for all $\ell \geq 0$ and $(t, x) \in \Sigma \times D_{r}$, we get

$$
\sum_{m \geq 0}\left|\partial_{t}^{\ell} w_{m}(t, x)\right| \leq C^{\prime} K^{\prime \ell} \Gamma(1+(s+1) \ell)
$$

In particular, for $\ell=0$, the series $\sum_{m \geq 0} w_{m}(t, x)$ is normally convergent on $\Sigma \times D_{r}$. Therefore, its sum $w(t, x)$ is well-defined and holomorphic on $\Sigma \times D_{r}$. This proves condition 1 of definition 2.3 if we choose for $\Sigma$ a sector bisected by $\theta$ and opening larger than $\pi s=\pi / k$. Note that such a choice is already possible due to the definition of proper subsector (see note 2 ).

For all $\ell \geq 1$, the series $\sum_{m \geq 0} \partial_{t}^{\ell} w_{m}(t, x)$ is also normally convergent on $\Sigma \times D_{r}$. Thereby, the series $\sum_{m \geq 0} w_{m}(t, x)$ can be derivated termwise infinitely many times with respect to $t$ and inequalities (4.13) imply

$$
\left|\partial_{t}^{\ell} w(t, x)\right| \leq C^{\prime} K^{\prime \ell} \Gamma(1+(s+1) \ell)
$$

for all $\ell \geq 0$ and $(t, x) \in \Sigma \times D_{r}$. This proves condition 3 of definition 2.3 (we consider here proper subsectors of $\Sigma$ ).

Note that the fact that all derivatives of $w(t, x)$ with respect to $t$ are bounded on $\Sigma$ implies the existence of $\lim _{t \rightarrow 0} \partial_{t}^{\ell} w(t, x)$ for all $x \in D_{r}$ and thereby the $\underset{t \rightarrow 0}{t \in \Sigma}$

existence of the Taylor series of $w$ at 0 on $\Sigma$ for all $x \in D_{r}$ (see for instance [20, Cor. 1.1.3.3]; see also [15, Prop. 2.2.11]). On the other hand, considering recurrence relations (4.4) with $w_{m}$ and the $k$-sum $g(t, x)$ instead of $\widetilde{w}_{m}$ and $\widetilde{g}(t, x)$, it is clear that $w(t, x)$ satisfies equation (4.3) with right-hand side $g(t, x)$ in place of $\widetilde{g}(t, x)$ and, consequently, so does its Taylor series. Then, since equation (4.3) has a unique formal series solution $\widetilde{w}(t, x)$ (see theorem 1.2 by exchanging the roles of $x$ and $t$ ), we then conclude that the Taylor expansion of $w(t, x)$ is $\widetilde{w}(t, x)$, which proves condition 2 of definition 2.3 .

This achieves the proof of the $k$-summability of $\widetilde{w}(t, x)$. Hence, the sufficient condition of point 1 of theorem 4.2, which ends the proof.

\section{References}

[1] W. Balser. Divergent solutions of the heat equation: on an article of Lutz, Miyake and Schäfke. Pacific J. Math., 188(1):53-63, 1999.

[2] W. Balser. Formal power series and linear systems of meromorphic ordinary differential equations. Universitext. Springer-Verlag, New-York, 2000.

[3] W. Balser. Multisummability of formal power series solutions of partial differential equations with constant coefficients. J. Differential Equations, 201(1):63-74, 2004. 
[4] W. Balser, B. J. L. Braaksma, J.-P. Ramis, and Y. Sibuya. Multisummability of formal power series solutions of linear ordinary differential equations. Asymptot. Anal., 5(1):27-45, 1991.

[5] W. Balser and M. Loday-Richaud. Summability of solutions of the heat equation with inhomogeneous thermal conductivity in two variables. $A d v$. Dyn. Syst. Appl., 4(2):159-177, 2009.

[6] W. Balser and M. Miyake. Summability of formal solutions of certain partial differential equations. Acta Sci. Math. (Szeged), 65(3-4):543-551, 1999.

[7] W. Balser and M. Yoshino. Gevrey order of formal power series solutions of inhomogeneous partial differential equations with constant coefficients. Funkcial. Ekvac., 53:411-434, 2010.

[8] B. L. J. Braaksma. Multisummability and Stokes multipliers of linear meromorphic differential equations. J. Differential Equations, 92:45-75, 1991

[9] M. Canalis-Durand, J.-P. Ramis, R. Schäfke, and Y. Sibuya. Gevrey solutions of singularly perturbed differential equations. J. Reine Angew. Math., 518:95-129, 2000.

[10] O. Costin, H. Park, and Y. Takei. Borel summability of the heat equation with variable coefficients. J. Differential Equations, 252(4):3076-3092, 2012.

[11] M. Hibino. Borel summability of divergence solutions for singular first-order partial differential equations with variable coefficients. I. J. Differential Equations, 227(2):499-533, 2006.

[12] M. Hibino. On the summability of divergent power series solutions for certain first-order linear pdes. Opuscula Math., 35(5):595-624, 2015.

[13] K. Ichinobe. On $k$-summability of formal solutions for a class of partial differential operators with time dependent coefficients. J. Differential Equations, 257(8):3048-3070, 2014.

[14] M. Loday-Richaud. Stokes phenomenon, multisummability and differential Galois groups. Ann. Inst. Fourier (Grenoble), 44(3):849-906, 1994.

[15] M. Loday-Richaud. Divergent series and differential equations. Prépublication du LAREMA, 375, 2014.

[16] D. A. Lutz, M. Miyake, and R. Schäfke. On the borel summability of divergent solutions of the heat equation. Nagoya Math. J., 154:1-29, 1999.

[17] S. Malek. On the summability of formal solutions of linear partial differential equations. J. Dyn. Control Syst., 11(3):389-403, 2005.

[18] S. Malek. On the Stokes phenomenon for holomorphic solutions of integrodifferential equations with irregular singularity. J. Dyn. Control Syst., 14(3):371-408, 2008. 
[19] S. Malek. Gevrey functions solutions of partial differential equations with fuchsian and irregular singularities. J. Dyn. Control Syst., 15(2):277-305, 2009.

[20] B. Malgrange. Sommation des séries divergentes. Expo. Math., 13:163-222, 1995.

[21] B. Malgrange and J.-P. Ramis. Fonctions multisommables. Ann. Inst. Fourier (Grenoble), 42:353-368, 1992.

[22] J. Martinet and J.-P. Ramis. Elementary acceleration and multisummability. Ann. Inst. H. Poincaré Phys. Théor., 54(4):331-401, 1991.

[23] S. Michalik. Multisummability of formal solutions of inhomogeneous linear partial differential equations with constant coefficients. J. Dyn. Control Syst., 18(1):103-133, 2012.

[24] M. Miyake. Newton polygons and formal Gevrey indices in the CauchyGoursat-Fuchs type equations. J. Math. Soc. Japan, 43(2):305-330, 1991.

[25] M. Miyake. Borel summability of divergent solutions of the Cauchy problem to non-Kovaleskian equations. In Partial differential equations and their applications (Wuhan, 1999), pages 225-239. World Sci. Publ., River Edge, N.J, 1999 .

[26] M. Nagumo. Über das Anfangswertproblem partieller Differentialgleichungen. Jap. J. Math., 18:41-47, 1942.

[27] S. Ouchi. Multisummability of formal solutions of some linear partial differential equations. J. Differential Equations, 185(2):513-549, 2002.

[28] S. Ouchi. Borel summability of formal solutions of some first order singular partial differential equations and normal forms of vector fields. J. Math. Soc. Japan, 57(2):415-460, 2005.

[29] M. E. Pliś and B. Ziemian. Borel resummation of formal solutions to nonlinear Laplace equations in 2 variables. Ann. Polon. Math., 67(1):31-41, 1997.

[30] J.-P. Ramis. Les séries $k$-sommables et leurs applications. In Complex analysis, microlocal calculus and relativistic quantum theory (Proc. Internat. Colloq., Centre Phys., Les Houches, 1979), volume 126 of Lecture Notes in Phys., pages 178-199. Springer, Berlin, 1980.

[31] J.-P. Ramis. Théorèmes d'indices Gevrey pour les équations différentielles ordinaires. Mem. Amer. Math. Soc., 48:viii+95, 1984.

[32] P. Remy. Gevrey order and summability of formal series solutions of some classes of inhomogeneous linear partial differential equations with variable coefficients. J. Dyn. Control Syst., to appear.

[33] H. Tahara and H. Yamazawa. Multisummability of formal solutions to the cauchy problem for some linear partial differential equations. J. Differential Equations, 255(10):3592-3637, 2013. 
[34] A. Yonemura. Newton polygons and formal Gevrey classes. Publ. Res. Inst. Math. Sci., 26:197-204, 1990. 